PSYCHOMETRIKA-VOL. 69, NO. 3, 375-399

SEPTEMBER 2004

\title{
TUCKER2 HIERARCHICAL CLASSES ANALYSIS
}

\author{
Eva Ceulemans and Iven Van Mechelen \\ KATHOLIEKE UNIVERSITEIT LEUVEN
}

\begin{abstract}
This paper presents a new hierarchical classes model, called Tucker2-HICLAS, for binary threeway three-mode data. As any three-way hierarchical classes model, the Tucker2-HICLAS model includes a representation of the association relation among the three modes and a hierarchical classification of the elements of each mode. A distinctive feature of the Tucker2-HICLAS model, being closely related to the Tucker3-HICLAS model (Ceulemans, Van Mechelen \& Leenen, 2003), is that one of the three modes is minimally reduced and, hence, that the differences among the association patterns of the elements of this mode are maximally retained in the model. Moreover, as compared to Tucker3-HICLAS, Tucker2HICLAS implies three rather than four different types of parameters and as such is simpler to interpret. Two types of Tucker2-HICLAS models are distinguished: a disjunctive and a conjunctive type. An algorithm for fitting the Tucker2-HICLAS model is described and evaluated in a simulation study. The model is illustrated with longitudinal data on interpersonal emotions.
\end{abstract}

Key words: three-way three-mode data, binary data, hierarchical classes, multiway data analysis, clustering.

\section{Introduction}

Three-way three-mode data are often found in psychology. Examples include the scores of a number of children on various items measured at different ages, data on the strength of different emotions as elicited by various situations in different cultures, and ratings by a number of clinicians on the intensity of various symptoms for a number of patients. In this paper, we will refer to the elements of the first mode as objects, to the elements of the second mode as attributes, and to the elements of the third mode as sources.

To model real-valued three-way three-mode data, one may consider a family of models that generalizes standard two-way two-mode principal component analysis (PCA); this family includes the PARAFAC/CANDECOMP, Tucker 3 and Tucker2 models. The Tucker3 model (Tucker, 1966) reduces each mode of a data array to a few components, where the number of components may differ across modes, and describes the interactions among the components of the three modes in a small three-way three-mode core array. Formally, the Tucker 3 model approximates an $I \times J \times K$ data array $\underline{\mathbf{D}}$ by an $I \times J \times K$ model array $\underline{\mathbf{M}}$ that can be decomposed into component matrices $\mathbf{A}(I \times P), \mathbf{B}(J \times Q), \mathbf{C}(K \times R)$ and a core array $\underline{\mathbf{G}}(P \times Q \times R)$ :

$$
d_{i j k} \approx m_{i j k}=\sum_{p=1}^{P} \sum_{q=1}^{Q} \sum_{r=1}^{R} a_{i p} b_{j q} c_{k r} g_{p q r}
$$

The first author is a Researcher of the Fund for Scientific Research-Flanders (Belgium). The research reported in this paper was partially supported by the Research Council of K.U. Leuven (GOA/2000/02). The authors are grateful to Iwin Leenen for the fruitful discussions. Correspondence concerning this paper should be addressed to Eva Ceulemans, Department of Psychology, Tiensestraat 102, B-3000 Leuven, Belgium. Email: Eva.Ceulemans@psy.kuleuven.ac.be. 
The PARAFAC/CANDECOMP model is a constrained Tucker 3 model in that the core array takes the form of a superidentity array (Carroll \& Chang, 1970; Harshman, 1970), implying that the model formula can be rewritten as:

$$
d_{i j k} \approx m_{i j k}=\sum_{r=1}^{R} a_{i r} b_{j r} c_{k r} .
$$

Yet, for various three-way three-mode data sets, reducing all three modes is not desirable. Examples include longitudinal data, for which one typically may not want to reduce the time mode (see also Kroonenberg \& De Leeuw, 1980). This has been one of the major reasons for developing the Tucker 2 model. The latter model reduces only two of the three modes to components; the core array then represents the interactions among the elements of the free mode and the components of the reduced modes (Kroonenberg \& De Leeuw, 1980; Kroonenberg, 1983). Formally, the Tucker2 model can be written as

$$
d_{i j k} \approx m_{i j k}=\sum_{p=1}^{P} \sum_{q=1}^{Q} a_{i p} b_{j q} g_{p q k},
$$

with component matrices $\mathbf{A}(I \times P), \mathbf{B}(J \times Q)$ and a core array $\underline{\mathbf{G}}(P \times Q \times K)$. Although in most cases the Tucker 2 model includes more parameters than the Tucker 3 model, Tucker 2 is conceptually simpler to grasp. Like the much more restrictive PARAFAC/CANDECOMP model, Tucker 2 implies three types of parameters (i.e., $a_{i p}, b_{j q}$, and $g_{p q k}$ ), whereas with Tucker 3 four types have to be handled (i.e., $a_{i p}, b_{j q}, c_{k r}$, and $g_{p q r}$ ).

For the representation of binary $N$-way $N$-mode data, one may consider the family of hierarchical classes (HICLAS) models (De Boeck \& Rosenberg, 1988; Leenen, Van Mechelen, De Boeck, \& Rosenberg, 1999; Van Mechelen, De Boeck, \& Rosenberg, 1995). An attractive feature of the hierarchical classes models is that they not only imply a decomposition rule but also represent relations of equivalence and hierarchy among the elements of each mode. Within the HICLAS family, Leenen et al. (1999) and Ceulemans et al. (2003) recently proposed the INDCLAS and Tucker3-HICLAS models for binary three-way three-mode data. These models may be considered the hierarchical classes equivalents of the PARAFAC/CANDECOMP and Tucker3 models respectively. As such, the Tucker3-HICLAS model reduces each mode to a set of a few binary variables, called bundles, and represents the relation among the three sets of bundles in a binary three-way three-mode core array. In addition, the bundles are restricted to represent the relations of equivalence and hierarchy in each of the three modes, yielding a hierarchical classification of each mode.

When fitting a hierarchical classes model to binary three-way three-mode data, one may also wish to reduce only two of the three modes of the data set for the very same reasons that gave rise to the development of the Tucker2 model: Firstly, for some data sets one may prefer an unreduced modeling of one mode in terms of its association with the two other modes implied by the data. Secondly, Tucker3-HICLAS implies four different types of parameters, which one may wish to decrease in order to simplify the model interpretation. In this paper, we therefore present the hierarchical classes counterpart of the Tucker 2 model, which will be called the Tucker2 hierarchical classes model (Tucker2-HICLAS).

The remainder of this paper is organized in five sections: Section 2 briefly recapitulates the Tucker3-HICLAS model and introduces the new Tucker2-HICLAS model. Two types of the Tucker2-HICLAS model will further be considered: a disjunctive and a conjunctive type. Section 3 describes an algorithm for fitting a Tucker2-HICLAS model to a data array. In Section 4, the performance of the algorithm is evaluated in a simulation study. In Section 5, the Tucker2HICLAS model is illustrated by application to interpersonal emotion data. A final section contains some concluding remarks. 


\section{Theory}

\subsection{The Disjunctive Tucker3-HICLAS Model}

\subsubsection{Specification of the Model}

A rank $(P, Q, R)$ Tucker3-HICLAS model approximates an $I \times J \times K$ object by attribute by source binary data array $\underline{\mathbf{D}}$ by an $I \times J \times K$ binary model array $\underline{\mathbf{M}}$ that can be decomposed into an $I \times P$ binary matrix $\mathbf{A}$, a $J \times Q$ binary matrix $\mathbf{B}$, a $K \times R$ binary matrix $\mathbf{C}$, and a $P \times Q \times R$ binary array $\underline{\mathbf{G}}$. The columns of $\mathbf{A}, \mathbf{B}$, and $\mathbf{C}$ define $P, Q$, and $R$ (possibly overlapping) clusters, called bundles, of objects, attributes and sources, respectively; $\mathbf{A}, \mathbf{B}$, and $\mathbf{C}$ are therefore called object, attribute, and source bundle matrices. $\underline{\mathbf{G}}$ represents the ternary association relation among the three sets of bundles and is called a core array.

As with all hierarchical classes models, the Tucker3-HICLAS model represents three types of structural relations in $\underline{\mathbf{M}}$ : association, equivalence and hierarchy.

Association. The association relation is the ternary relation between the objects, attributes, and sources as defined by the 1-entries of the array $\underline{\mathbf{M}}$. The disjunctive Tucker3-HICLAS model represents the association relation by the following association rule:

$$
m_{i j k}=\bigoplus_{p=1}^{P} \bigoplus_{q=1}^{Q} \bigoplus_{r=1}^{R} a_{i p} b_{j q} c_{k r} g_{p q r},
$$

where $\bigoplus$ denotes the Boolean sum. Equation (4) can be reformulated as

$$
m_{i j k}=1 \Leftrightarrow \exists p, q, r: a_{i p}=1 \wedge b_{j q}=1 \wedge c_{k r}=1 \wedge g_{p q r}=1 .
$$

The latter implies that an object $i$, an attribute $j$, and a source $k$ are associated in $\underline{\mathbf{M}}$ iff an object bundle, an attribute bundle, and a source bundle exist, to which $i, j$ and $k$ respectively belong, and that are associated in $\underline{\mathbf{G}}$. Because of the existential quantifier, (5) can be considered a disjunctive association rule.

Equivalence. Three equivalence relations are defined, one on each mode of $\underline{\mathbf{M}}$. In the case of the object mode, object $i$ is equivalent to object $i^{\prime}$ in $\underline{\mathbf{M}}$ iff they are associated with the same (attribute, source) pairs in $\underline{\mathbf{M}}$. Equivalent objects constitute an object class. In the disjunctive Tucker3-HICLAS model, the equivalence relation among the objects is represented in the object bundle matrix $\mathbf{A}$ in that equivalent objects have identical bundle patterns (i.e., they belong to the same set of object bundles). The equivalence relations among the attributes and sources are defined and represented similarly.

Hierarchy. On each of the three modes of $\underline{\mathbf{M}}$ a hierarchical relation is defined. In the case of the object mode, object $i$ is hierarchically below object $i^{\prime}$ in $\underline{\mathbf{M}}$ iff the set of (attribute, source) pairs associated with $i$ constitutes a proper subset of the set of (attribute, source) pairs associated with $i^{\prime}$. In the disjunctive Tucker3-HICLAS model, this hierarchical relation is represented in the object bundle matrix $\mathbf{A}$ in that object $i$ is hierarchically below object $i^{\prime}$ iff the bundle pattern of $i$ is a proper subset of the bundle pattern of $i^{\prime}$. The hierarchical relations among the attributes and sources are defined and represented similarly. As an aside, we note that from a bundle matrix one may also derive equivalence relations between an element and disjunctive combinations of other elements: If the bundle patterns of two (or more) hierarchically lower elements $j$ and $k$ span the bundle pattern of a hierarchically higher element $i$, association with $i$ is equivalent to association with $j$ or $k$.

\subsubsection{Variants}

As for all hierarchical classes models, one may distinguish several variants of the disjunctive Tucker3-HICLAS model. In particular, 16 variants of the disjunctive Tucker3-HICLAS model 
can be distinguished, the association rules of which are obtained by replacing any of the arrays $\mathbf{A}$, $\mathbf{B}, \mathbf{C}$, or $\underline{\mathbf{G}}$ in the association rule (5) by their Boolean complement. The variants may therefore be denoted by labels of the form " ${ }^{c} 2^{c} 3 \mathrm{G}$," with 1, 2, 3, and G denoting $\mathbf{A}, \mathbf{B}, \mathbf{C}$, and $\underline{\mathbf{G}}$, respectively, and ${ }^{c}$ indicating that the array in question was replaced by its Boolean complement. For example, the variant defined by (5) has label "123G."

Note that these 16 different types of disjunctive Tucker3-HICLAS models must be considered reparametrizations and, hence, mathematically equivalent model types; as such, the existence of 16 different variants has nothing to do with any lack of identifiability of the " $123 \mathrm{G}$ " variant described in Subsection 2.1.1. The 16 variants may bear different interpretations, though, implying that the choice between them should depend on substantive considerations only (see also Van Mechelen et al., 1995; Leenen et al., 1999).

\subsubsection{Existence and Uniqueness}

With respect to existence, extending the proof by De Boeck and Rosenberg (1988), it is straightforward to show that for each $I \times J \times K$ model array $\underline{\mathbf{M}}$, a $(P, Q, R)$ Tucker3-HICLAS model exists (with $(P, Q, R) \leq(I, J, K)$ ).

The issue of (non)uniqueness can be considered on three levels: (1) general nonuniqueness, which holds for all Tucker3-HICLAS decompositions irrespective of the model array at hand; (2) model-driven nonuniqueness: for a specific model array $\underline{\mathbf{M}}$, several Tucker3-HICLAS decompositions may exist; and (3) data-driven nonuniqueness: for a specific data array $\underline{\mathbf{D}}$, several model arrays $\underline{\mathbf{M}}$ and associated decompositions may exist that approximate the data (almost) equally well. In this paper, we focus on the first two types of nonuniqueness.

Regarding general nonuniqueness, for any Tucker3-HICLAS decomposition it holds that the three sets of bundles can be permuted. Note that permutational freedom is the hierarchical classes equivalent of rotational freedom in principal component analysis. ${ }^{1}$ Regarding modeldriven uniqueness, Ceulemans and Van Mechelen (2003) proved the following sufficient condition for the uniqueness of a Tucker3-HICLAS decomposition: If a $(P, Q, R)$ Tucker3-HICLAS decomposition of an $I \times J \times K$ binary array $\underline{\mathbf{M}}$ exists, such that (1) all bundle-specific classes (i.e., classes of elements who belong to one bundle only) of $\mathbf{A}, \mathbf{B}$, and $\mathbf{C}$ are nonempty, and (2) no object (resp. attribute, source) plane of $\mathbf{G}$ is a subset of the Boolean sum of the other object (resp. attribute, source) planes of $\underline{\mathbf{G}}$, this decomposition is unique upon a permutation of the object, attribute, and source bundles.

\subsection{The Disjunctive Tucker2-HICLAS Model}

\subsubsection{Specification of the Model}

The key difference between the Tucker3- and Tucker2-HICLAS models is that Tucker2HICLAS reduces only two of the three modes to a few bundles. More specifically, a $(P, Q)$ Tucker2-HICLAS model implies a decomposition of an $I \times J \times K$ binary model array $\underline{\mathbf{M}}$ into an $I \times P$ binary object bundle matrix $\mathbf{A}$, a $J \times Q$ binary attribute bundle matrix $\mathbf{B}$, and a $P \times Q \times K$ binary core array $\underline{\mathbf{G}}$.

Since the definitions of the three structural relations are identical for Tucker3-HICLAS and Tucker2-HICLAS, only the representation of the latter relations will be discussed here in detail. The hypothetical applicant by judicial job by rater array $\underline{\mathbf{M}}$ in Table 1 (with a 1 in cell $i j k$ iff rater $k$ finds applicant $i$ suitable for judicial job $j$, and 0 otherwise) and the associated disjunctive

\footnotetext{
${ }^{1}$ This implies that the use of rotational criteria like VARIMAX in principal component analysis is not the counterpart of the use of the criterion of set-theoretical consistency with Boolean models; indeed, set-theoretical consistency pertains to model-driven rather than general nonuniqueness (see also Van Mechelen et a1., 1995).
} 
TABLE 1

Hypothetical binary applicant by judicial job by rater job suitability data array

\begin{tabular}{|c|c|c|c|c|c|c|c|c|c|c|c|c|c|c|c|c|}
\hline \multirow[b]{2}{*}{ Raters } & \multicolumn{4}{|c|}{ Diane } & \multicolumn{4}{|c|}{ Judy } & \multicolumn{4}{|c|}{ Will } & \multicolumn{4}{|c|}{ Jack } \\
\hline & $\stackrel{\vec{\Omega}}{\Xi}$ & 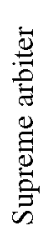 & $\begin{array}{l}5 \\
\stackrel{5}{0} \\
0 \\
0 \\
0 \\
0 \\
0 \\
0 \\
0 \\
0 \\
0 \\
0 \\
0\end{array}$ & $\frac{\dot{0}}{\frac{0}{0}}$ & $\stackrel{\vec{\Omega}}{\Xi}$ & 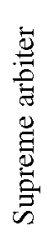 & 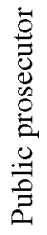 & $\frac{\overrightarrow{0}}{\frac{0}{0}}$ & $\stackrel{\underline{\Omega}}{\Xi}$ & 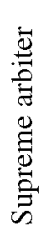 & 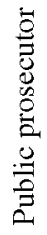 & $\frac{1}{0}$ & $\stackrel{\vec{\Xi}}{\Xi}$ & 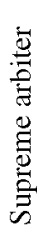 & 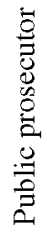 & 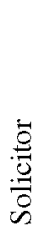 \\
\hline$A$ & 1 & 1 & 0 & 0 & 1 & 1 & 0 & 0 & 0 & 0 & 0 & 0 & 0 & 0 & 0 & 0 \\
\hline$B$ & 0 & 0 & 0 & 0 & 1 & 1 & 0 & 0 & 1 & 1 & 0 & 0 & 1 & 1 & 0 & 0 \\
\hline$C$ & 1 & 1 & 0 & 0 & 1 & 1 & 1 & 1 & 1 & 0 & 1 & 1 & 1 & 0 & 1 & 1 \\
\hline$D$ & 0 & 0 & 0 & 0 & 1 & 0 & 1 & 1 & 1 & 0 & 1 & 1 & 1 & 0 & 1 & 1 \\
\hline$E$ & 0 & 0 & 0 & 0 & 1 & 1 & 1 & 1 & 1 & 1 & 1 & 1 & 1 & 1 & 1 & 1 \\
\hline$F$ & 1 & 1 & 0 & 0 & 1 & 1 & 0 & 0 & 1 & 1 & 0 & 0 & 1 & 1 & 0 & 0 \\
\hline$G$ & 1 & 1 & 0 & 0 & 1 & 1 & 0 & 0 & 0 & 0 & 0 & 0 & 0 & 0 & 0 & 0 \\
\hline
\end{tabular}

$(2,2)$ Tucker2-HICLAS model in Table 2 serve as guiding examples. Given the bundle matrices in Table 2, assume that, based on external information regarding the applicants, the applicant bundles can be interpreted as "Makes fair decisions" and "Has profound knowledge of legal history"; the job bundles can be interpreted as "Bench jobs" and "Attorney jobs."

Association. The disjunctive Tucker2-HICLAS model represents the association relation by the following rule:

$$
m_{i j k}=\bigoplus_{p=1}^{P} \bigoplus_{q=1}^{Q} a_{i p} b_{j q} g_{p q k} .
$$

Rule (6) is equivalent to

$$
m_{i j k}=1 \Leftrightarrow \exists p, q: a_{i p}=1 \wedge b_{j q}=1 \wedge g_{p q k}=1 .
$$

In our example, (7) could be interpreted as follows: Rater $k$ will find applicant $i$ suitable for job $j$ iff the applicant has at least one of the qualities $p\left(a_{i p}=1\right)$ that the rater deems sufficient for a job

TABLE 2.

\begin{tabular}{|c|c|c|c|c|c|c|c|c|c|c|}
\hline \multicolumn{6}{|c|}{ Bundle matrices } & \multicolumn{5}{|c|}{ Core array } \\
\hline \multirow[b]{2}{*}{ Applicants } & \multicolumn{2}{|c|}{$\begin{array}{l}\text { Applicant } \\
\text { bundles }\end{array}$} & \multirow[b]{2}{*}{ Jobs } & \multicolumn{2}{|c|}{$\begin{array}{c}\text { Job } \\
\text { bundles }\end{array}$} & \multirow[b]{2}{*}{ Raters } & \multicolumn{2}{|c|}{$J B_{1}$} & \multicolumn{2}{|c|}{$J B_{2}$} \\
\hline & $A B_{1}$ & $A B_{2}$ & & $J B_{1}$ & $J B_{2}$ & & $A B_{1}$ & $A B_{2}$ & $A B_{1}$ & $A B_{2}$ \\
\hline Diane & 1 & 0 & Jurist & 1 & 1 & $A$ & 1 & 0 & 0 & 0 \\
\hline Judy & 1 & 1 & Supreme arbiter & 1 & 0 & $B$ & 0 & 1 & 0 & 0 \\
\hline Will & 0 & 1 & Public prosecutor & 0 & 1 & $C$ & 1 & 0 & 0 & 1 \\
\hline \multirow[t]{4}{*}{ Jack } & 0 & 1 & Solicitor & 0 & 1 & $D$ & 0 & 0 & 0 & 1 \\
\hline & & & & & & $E$ & 0 & 1 & 0 & 1 \\
\hline & & & & & & $F$ & 1 & 1 & 0 & 0 \\
\hline & & & & & & $G$ & 1 & 0 & 0 & 0 \\
\hline
\end{tabular}

Disjunctive Tucker2-HICLAS model for the array of Table 1 
type $q\left(g_{p q k}=1\right)$ to which the job belongs $\left(b_{j q}=1\right)$. The latter means that from the core array one may derive the implicit rules of the seven raters for job suitability, as $\underline{\mathbf{G}}$ indicates separately for each rater which applicant qualities are deemed singly sufficient for ensuring suitability for the different job types. For example, from the model in Table 2, it can be derived that Rater $B$ considers "Profound knowledge of legal history" a singly sufficient quality to appoint someone for a "Bench job"; furthermore, as this quality is the only sufficient one for this rater, it is also necessary. Therefore, only "Diane" is unsuitable for the job of "Supreme arbiter" according to Rater $B$, because she and only she does not meet the requirement. Note that ( 7 ) is a disjunctive rule, as only one combination of a quality and a job type has to satisfy the given condition.

Equivalence. In the disjunctive Tucker2-HICLAS model, equivalent objects and attributes have identical bundle patterns, whereas equivalent sources have identical core planes. For example, Raters $A$ and $G$ are equivalent in Table 1; hence, they have identical core planes in Table 2.

Hierarchy. The disjunctive Tucker2-HICLAS model represents the hierarchical relations among the elements in terms of subset-superset relations between either bundle patterns or core planes. For example, in Table 1, "Public prosecutor" is hierarchically below "Jurist"; hence, the bundle pattern of "Public prosecutor" is a proper subset of the bundle pattern of "Jurist" in Table 2. As an aside, we note that from the bundle matrices and core array one may also derive equivalence relations between an element and disjunctive combinations of other elements: If the bundle patterns (resp. core planes) of two (or more) hierarchically lower elements $j$ and $k$ span the bundle pattern (resp. core plane) of a hierarchically higher element $i$, association with $i$ is equivalent to association with $j$ or $k$; that is, $i$ is equivalent to the disjunction of $j$ and $k$. For example, in Table 2, being rated suitable for "Jurist" is equivalent to being rated suitable for "Supreme arbiter" or for "Public prosecutor," because the bundle patterns of "Supreme arbiter" and "Public prosecutor" span the bundle pattern of "Jurist." The latter makes sense from a substantive point of view as a jurist may act either as judge or as attorney.

\subsubsection{Variants}

Taking Boolean complements of any of the matrices $\mathbf{A}, \mathbf{B}$, or $\underline{\mathbf{G}}$, the association rules of eight mathematically equivalent variants of the disjunctive Tucker2-HICLAS model are obtained. One may denote a Tucker2-HICLAS variant under consideration by a label of the form " $13^{c} \mathrm{G}$," with the two integers indicating which two modes are reduced. For example, the variant specified by (7) has label " $12 \mathrm{G}$. ",

\subsubsection{Existence and Uniqueness}

Regarding existence, it can be easily shown that for each $I \times J \times K$ model array $\underline{\mathbf{M}}$, a perfect $(P, Q)$ Tucker2-HICLAS model exists (with $(P, Q) \leq(I, J)$ ).

Regarding (non)uniqueness, as for Tucker3-HICLAS (see Subsection 2.1.3), one may distinguish between general, model-driven, and data-driven nonuniqueness. In this paper, we only consider the first two types of nonuniqueness in more detail. With respect to general nonuniqueness, for each Tucker2-HICLAS decomposition, it holds that the two sets of bundles may be permuted. With respect to model-driven nonuniqueness, we proved (see Appendix) the following sufficient condition for the uniqueness of a disjunctive Tucker2-HICLAS decomposition: If a $(P, Q)$ disjunctive Tucker2-HICLAS decomposition of an $I \times J \times K$ binary array $\underline{\mathbf{M}}$ exists, such that (1) all bundle-specific classes of $\mathbf{A}$ and $\mathbf{B}$ are nonempty and (2) no object (resp. attribute) plane of $\underline{\mathbf{G}}$ is a subset of the Boolean sum of the other object (resp. attribute) planes of $\underline{\mathbf{G}}$, this decomposition is unique upon a permutation of the object and attribute bundles. One may conclude that the conditions for a Tucker2-HICLAS model to be unique do not differ considerably in strength from those for the other three-way HICLAS models (Tucker3-HICLAS and INDCLAS; 
see Ceulemans \& Van Mechelen, 2003), unlike the case for the real-valued counterparts of the models in question. Furthermore, one will note in the Simulation study reported in Section 4 that the restrictiveness of the specified condition is evaluated by (1) calculating the proportion of randomly generated true models that satisfy this condition and (2) investigating the extent to which this proportion differs as a function of the size and the rank of the true model.

\subsubsection{Graphical Representation}

The disjunctive Tucker2-HICLAS model can be given an overall graphical representation that accounts for the three types of structural relations in the model. Figure 1 shows the graphical representation of the Tucker2-HICLAS model in Table 2. The object hierarchy shows up in the upper half of the representation. Equivalent objects are enclosed by boxes, representing the object classes, and the hierarchical relations between the object classes are indicated by lines between the respective boxes. Similarly, the attribute hierarchy shows up upside down in the lower half of the representation. The object and attribute hierarchies are further linked by paths between the base classes of the object and attribute bundles that are associated in $\underline{\mathbf{G}}$. The hexagons included in the paths indicate for which sources the respective associations hold. For example, $A B_{2}$ "Has a profound knowledge of legal history" and $J B_{1}$ "Bench job" are associated according to Raters $B, E$, and $F$ in $\underline{\mathbf{G}}$; hence, a path exists between the base classes of $A B_{2}$ and $J B_{1}$, with the hexagon containing Raters $B, E$ and $F$.

From the overall graphical representation, the association relation among objects, attributes, and sources can be read as follows: An object $i$ is associated with attribute $j$ according to source $k$ iff a downward path exists from object $i$ to attribute $j$ that includes source $k$. For example, "Will" is suitable for the job of "Supreme arbiter" according to Raters $B, E$, and $F$, given that a path exists between "Will" and "Supreme arbiter" that goes via $B, E$, and $F$. Unlike those for objects and attributes, the equivalence and hierarchical relations among sources are not immediately visible in the representation. Therefore, one may consider drawing a separate picture of

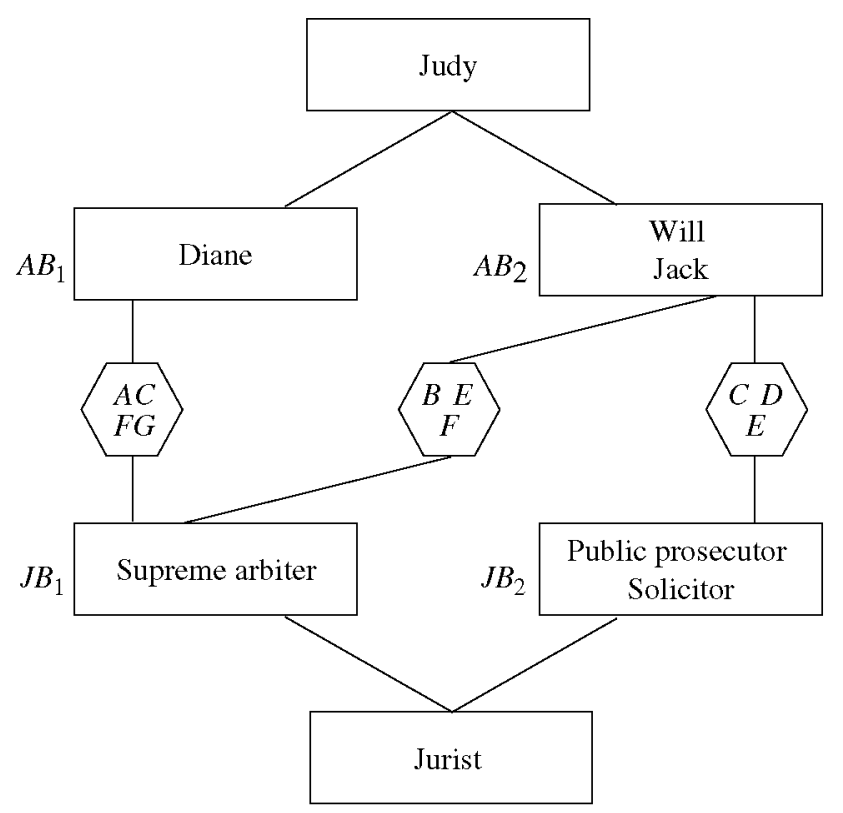

FIGURE 1.

Overall graphical representation of the disjunctive Tucker2-HICLAS model in Table 2. 


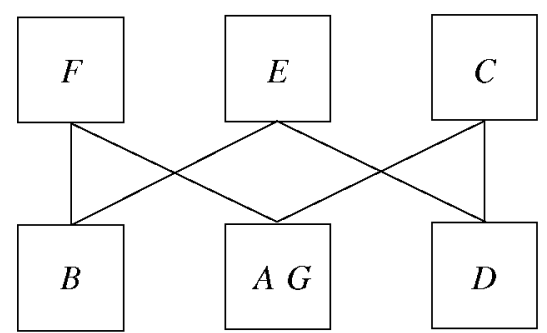

FTGURE 2.

Rater structure of the disjunctive Tucker2-HICLAS model in Table 2.

the source hierarchy. Figure 2 represents the rater structure of the disjunctive Tucker2-HICLAS model in Table 2.

\subsubsection{Relationship Between Disjunctive Tucker2-HICLAS and Disjunctive Tucker3-HICLAS}

Regarding the relationship between disjunctive Tucker2- and Tucker3-HICLAS, we first derive that each $(P, Q)$ Tucker2-HICLAS model is a $(P, Q, R)$ Tucker3-HICLAS model, with $R \leq \min (K, P Q)$. In order to see this, consider the $P \times Q \times K$ Tucker2-HICLAS core array $\underline{\mathbf{G}}$ as well as its $P Q \times K$ matricization $\tilde{\mathbf{G}}$. Assume, without loss of generality, that $\tilde{\mathbf{G}}$ has Schein rank $R^{*}$; the latter means that $R^{*}$ is the smallest integer such that $\tilde{\mathbf{G}}$ can be decomposed into a $P Q \times R^{*}$ matrix $\mathbf{G}^{*}$ and a $K \times R^{*}$ matrix $\mathbf{C}$ :

$$
\tilde{g}_{p q k}=\bigoplus_{r=1}^{R^{*}} g_{p q r}^{*} c_{k r} .
$$

Note that it necessarily holds that $R^{*} \leq \min (P Q, K)$ (Kim, 1982). If we reshape $\tilde{\mathbf{G}}$ and $\mathbf{G}^{*}$ into three-way arrays $\underline{\mathbf{G}}$ and $\underline{\mathbf{G}}^{\star},(8)$ implies that

$$
g_{p q k}=\bigoplus_{r=1}^{R^{*}} g_{p q r}^{\star} c_{k r}
$$

Substituting (9) in the Tucker2-HICLAS association rule (6), we obtain

$$
m_{i j k}=\bigoplus_{p=1}^{P} \bigoplus_{q=1}^{Q} a_{i p} b_{j q} \bigoplus_{r=1}^{R^{*}} g_{p q r}^{\star} c_{k r},
$$

which equals the Tucker3-HICLAS association rule.

Conversely, each $(P, Q, R)$ Tucker3-HICLAS model can be converted into a $(P, Q)$ Tucker2-HICLAS model. Indeed, the Tucker3-HICLAS decomposition rule (4) can be rewritten as

$$
m_{i j k}=\bigoplus_{p=1}^{P} \bigoplus_{q=1}^{Q} a_{i p} b_{j q} \tilde{g}_{p q k},
$$

with

$$
\tilde{g}_{p q k}=\bigoplus_{r=1}^{R} c_{k r} g_{p q r} .
$$


The fact that each $(P, Q)$ Tucker2-HICLAS model is a $(P, Q, R)$ Tucker3-HICLAS model with $R \leq \min (K, P Q)$ qualifies the statement that Tucker2-HICLAS implies a reduction of only two of the three modes of the data array: Tucker2-HICLAS implicitly entails a reduction of the free mode as well. Note, however, that Ceulemans et al. (2003) proved that if $\underline{\mathbf{M}}$ can be perfectly reconstructed from a $(P, Q, R)$ Tucker3-HICLAS model with $R>P Q$, then a perfectly fitting $\left(P, Q, R^{\prime}\right)$ Tucker3-HICLAS model also exists with $R^{\prime}=P Q$. The latter means that given a specific rank $(P, Q)$, no $(P, Q, R)$ Tucker3-HICLAS model exists that implies less of a reduction of the elements of the free mode of the $(P, Q)$ Tucker2-HICLAS model. The Tucker2-HICLAS model may therefore be considered to imply a minimal reduction of the elements of the free mode.

\subsection{The Conjunctive Tucker2-HICLAS Model}

\subsubsection{Specification of the Model}

Analogous to the conjunctive counterpart of the original two-way two-mode hierarchical classes model (Van Mechelen et al., 1995), conjunctive variants of the three-way three-mode hierarchical classes models can be formulated. Conjunctive hierarchical classes models can be considered for at least three reasons. First, a disjunctive model includes a disjunctive association rule: For example, in the case of the data in Table 1, the disjunctive Tucker2-HICLAS association rule indicates that a rater will find an applicant suitable, if the applicant has at least one of the qualities that the rater deems sufficient for at least one of the job types to which the job belongs. From a substantive viewpoint, a more plausible rule might indicate that a rater will only find an applicant suitable, if the applicant has all the qualities that the rater deems necessary for the job types to which the job belongs. The latter is a conjunctive association rule, as included in a conjunctive Tucker2-HICLAS model. Second, from a disjunctive model, one may derive equivalences between an element and disjunctive combinations of other elements. In some cases, however, it may also be desirable to look for equivalences between an element and conjunctive combinations of other elements. For example, one may wish to know whether suitability for job A is equivalent to suitability for job $\mathrm{B}$ and for job $\mathrm{C}$. The latter can be achieved through conjunctive modeling. Third, for a given binary data array and a given rank, the best fitting conjunctive model may yield a better approximation of $\underline{\mathbf{D}}$ than the best fitting disjunctive model (or vice versa). An example is the data array in Table 3 , which is used for illustration below. Given rank $(3,2)$,

TABLE 3.

Hypothetical binary applicant by business job by rater job suitability data array

\begin{tabular}{|c|c|c|c|c|c|c|c|c|c|c|c|c|c|c|c|c|c|c|c|c|c|c|c|c|}
\hline \multirow[b]{2}{*}{ Raters } & \multicolumn{4}{|c|}{ Martha } & \multicolumn{4}{|c|}{ Rose } & \multicolumn{4}{|c|}{ Bob } & \multicolumn{4}{|c|}{ Tom } & \multicolumn{4}{|c|}{ John } & \multicolumn{4}{|c|}{ Annie } \\
\hline & 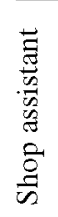 & 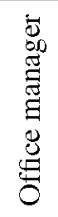 & 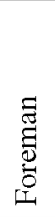 & 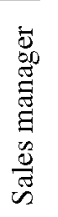 & 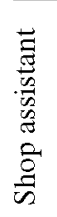 & 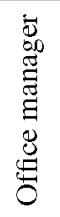 & 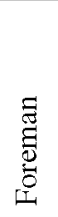 & 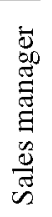 & 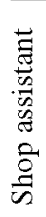 & 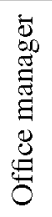 & $\begin{array}{l}\underset{\tilde{J}}{0} \\
\stackrel{0}{0} \\
\text { [1 }\end{array}$ & 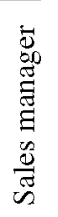 & 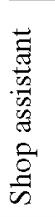 & 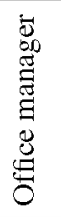 & 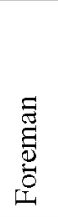 & 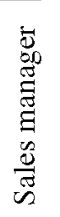 & 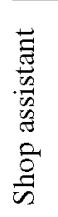 & 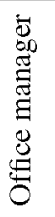 & 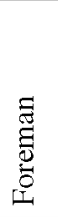 & 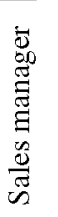 & 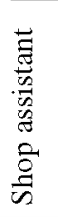 & 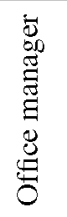 & 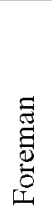 & 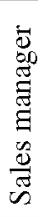 \\
\hline$A$ & 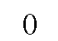 & 0 & 0 & 0 & 0 & 0 & 0 & 0 & 0 & 1 & 1 & 0 & 0 & 1 & 1 & 0 & 1 & 1 & 1 & 1 & 1 & 0 & 0 & $\Omega$ \\
\hline$B$ & 1 & 0 & 0 & 0 & 1 & 0 & 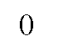 & ( & 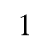 & 0 & & 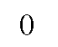 & & & 0 & & & & & & & 1 & & \\
\hline C & 0 & 0 & 0 & 0 & 0 & 0 & 0 & & 0 & 1 & 1 & 0 & & ( & 0 & 0 & 0 & 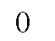 & 0 & 0 & 0 & 0 & & \\
\hline$D$ & 0 & 0 & 0 & 0 & 0 & ( & 0 & & 1 & 0 & & 0 & & & 0 & & & & & & & & & \\
\hline$E$ & 0 & 0 & 0 & 0 & 0 & 0 & 0 & ( & 0 & 1 & & 0 & 0 & & 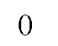 & 0 & & & & & & 0 & & \\
\hline$F$ & 0 & 0 & 0 & 0 & 0 & 0 & 0 & ( & 0 & 1 & 1 & 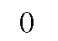 & 0 & & 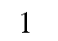 & 0 & 1 & 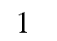 & 1 & & 1 & 0 & 0 & 0 \\
\hline$G$ & 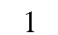 & 1 & 1 & 1 & 1 & 1 & 1 & 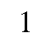 & 1 & 1 & 1 & 1 & & 0 & 0 & 0 & 0 & 0 & 0 & 0 & 0 & 0 & 0 & \\
\hline
\end{tabular}


a perfectly fitting conjunctive Tucker2-HICLAS model exists for these data, whereas the best fitting disjunctive Tucker2-HICLAS model implies $4.8 \%$ discrepancies with respect to the data.

Like the disjunctive Tucker2-HICLAS model, the conjunctive $(P, Q)$ Tucker2-HICLAS model implies a decomposition of an $I \times J \times K$ binary model array $\underline{\mathbf{M}}$ into an $I \times P$ binary object bundle matrix $\mathbf{A}$, a $J \times Q$ binary attribute bundle matrix $\mathbf{B}$, and a $P \times Q \times K$ binary core array $\underline{\mathbf{G}}$. As a guiding example, Table 4 presents a conjunctive $(3,2)$ Tucker2-HICLAS model for the hypothetical binary applicant by business job by rater data in Table 3 . Given the bundle matrices in Table 4, assume further that, drawing on external information regarding the applicants, the applicant bundles can be interpreted as "Holds a university degree," "Male," and "Prepared to work during weekends"; the job bundles can be labeled "Salesman" and "Executive function."

For our guiding example, a conjunctive Tucker2-HICLAS model may be considered that represents the three structural relations as follows:

Association. This relation is represented by the following rule:

$$
m_{i j k}=1 \Leftrightarrow \forall p, q: a_{i p}^{c}=0 \vee b_{j q}=0 \vee g_{p q k}=0 .
$$

Because of the universal quantifier $\forall$, (12) can be considered a conjunctive rule, as $\forall$ implies that the formulated condition has to hold for each (object bundle, attribute bundle) pair. Rule (12) is equivalent to

$$
m_{i j k}=1 \Leftrightarrow \forall q: b_{j q}=1 \Rightarrow\left(\forall p: g_{p q k}=1 \Rightarrow a_{i p}=1\right) .
$$

In our example in Table 3, rule (13) can be interpreted as follows: Rater $k$ will find applicant $i$ suitable for job $j$ iff, for any job type $q$ the job belongs to $\left(b_{j q}=1\right)$, the applicant has all the qualities $p\left(a_{i p}=1\right)$ that the rater deems necessary for that job type $\left(g_{p q k}=1\right)$. The latter implies that, in contrast to the core of the disjunctive Tucker2-HICLAS model, $\underline{\mathbf{G}}$ now indicates separately for each rater which applicant qualities are singly necessary and jointly sufficient for ensuring suitability for the different job types. For instance, it can be deduced from Table 4 that according to Rater $D$, "Male" and "Prepared to work during weekends" are singly necessary and jointly sufficient applicant qualities for an "Executive function." Therefore, only "John" is suitable for the function of "Foreman" according to Rater $D$, because he and only he meets the requirement. Note that if we define $K \times Q$ matrices $\tilde{\mathbf{A}}^{(i)}$ as follows

$$
\tilde{a}_{k q}^{(i)}=1 \Leftrightarrow\left(\forall p: g_{p q k}=1 \Rightarrow a_{i p}=1\right),
$$

\begin{tabular}{|c|c|c|c|c|c|c|c|c|c|c|c|c|c|}
\hline \multicolumn{7}{|c|}{ Bundle matrices } & \multirow[b]{3}{*}{ Raters } & \multicolumn{6}{|c|}{ Core array } \\
\hline \multirow[b]{2}{*}{ Applicants } & \multicolumn{3}{|c|}{$\begin{array}{l}\text { Applicant } \\
\text { bundles }\end{array}$} & \multirow[b]{2}{*}{ Jobs } & \multicolumn{2}{|c|}{$\begin{array}{c}\text { Job } \\
\text { bundles }\end{array}$} & & \multicolumn{3}{|c|}{$J B_{1}$} & \multicolumn{3}{|c|}{$J B_{2}$} \\
\hline & $A B_{1}$ & $A B_{2}$ & $A B_{3}$ & & $J B_{1}$ & $J B_{2}$ & & $A B_{1}$ & $A B_{2}$ & $A B_{3}$ & $A B_{1}$ & $A B_{2}$ & $A B_{3}$ \\
\hline Martha & 1 & 0 & 0 & Shop assistant & 1 & 0 & $A$ & 0 & 0 & 1 & 0 & 1 & 0 \\
\hline Rose & 1 & 0 & 0 & Office manager & 0 & 1 & $B$ & 0 & 0 & 0 & 0 & 0 & 1 \\
\hline Bob & 1 & 1 & 0 & Foreman & 0 & 1 & $C$ & 1 & 0 & 1 & 1 & 1 & 0 \\
\hline Tom & 0 & 1 & 0 & Sales manager & 1 & 1 & $D$ & 0 & 1 & 0 & 0 & 1 & 1 \\
\hline John & 0 & 1 & 1 & & & & $E$ & 0 & 0 & 1 & 1 & 1 & 0 \\
\hline \multirow[t]{2}{*}{ Annie } & 0 & 0 & 1 & & & & $F$ & 0 & 0 & 1 & 0 & 1 & 0 \\
\hline & & & & & & & $G$ & 1 & 0 & 0 & 1 & 0 & 0 \\
\hline
\end{tabular}

TABLE 4.

Conjunctive Tucker2-HICLAS model for the array of Table 3 
(13) can be rewritten as

$$
m_{i j k}=1 \Leftrightarrow \forall q: b_{j q}=1 \Rightarrow \tilde{a}_{k q}^{(i)}=1,
$$

which is the association rule of a conjunctive two-way HICLAS model (see Van Mechelen et al., 1995). The latter will turn out to be particularly useful for the graphical representation of the conjunctive Tucker2-HICLAS model.

Equivalence. In the conjunctive Tucker2-HICLAS model, equivalent objects and attributes once again have identical bundle patterns, whereas equivalent sources have identical core planes. For example, Raters $A$ and $F$ are equivalent in Table 3; hence, they have identical core planes in Table 4.

Hierarchy. In the conjunctive Tucker2-HICLAS model for our guiding example, the hierarchical relations among the objects are directly represented in $\mathbf{A}$, whereas the hierarchical relations among the attributes and sources are inversely represented in $\mathbf{B}$ and $\underline{\mathbf{G}}$. For example, in Table 3 Tom is hierarchically lower than John; consequently, Tom's bundle pattern is a proper subset of John's bundle pattern in Table 4. Also, "Sales manager" is hierarchically lower than "Foreman," implying that the bundle pattern of "Sales manager" is a proper superset of the bundle pattern of "Foreman." One may further derive from $\mathbf{A}^{c}$ (resp. B, $\underline{\mathbf{G}}$ ) equivalence relations between an object (resp. attribute, source) and conjunctive combinations of other objects (resp. attributes, sources): If the complemented bundle patterns (resp. bundle patterns, core planes) of two (or more) hierarchically higher objects (resp. attributes, sources) $j$ and $k$ span the complemented bundle pattern (resp. bundle pattern, core plane) of a hierarchically lower object (resp. attribute, source) $i$, association with $i$ is equivalent to association with $j$ and $k$; that is, $i$ is equivalent to the conjunction of $j$ and $k$. For example, from Table 4, it follows that suitability for "Sales manager" is equivalent to suitability for "Shop assistant" and for "Office manager," as the bundle patterns of "Shop assistant" and "Office manager" span the bundle pattern of "Sales manager."

\subsubsection{Variants}

As was the case for the disjunctive Tucker2-HICLAS model, eight mathematically equivalent variants of the conjunctive Tucker2-HICLAS model can be obtained by taking the Boolean complement of any of the matrices $\mathbf{A}, \mathbf{B}$, or $\underline{\mathbf{G}}$. The conjunctive variants can be labeled similar to the disjunctive variants (see 2.2.2). For example, the variant specified by association rule (12) is indicated by the label " $1{ }^{c} 2 \mathrm{G}$. ."

\subsubsection{Relation Between Disjunctive and Conjunctive Tucker2-HICLAS Models}

The disjunctive and conjunctive Tucker2-HICLAS models are dual models for given $P$ and $Q$. In particular, the arrays $\mathbf{A}, \mathbf{B}$, and $\underline{\mathbf{G}}$ constitute a disjunctive " $12 \mathrm{G}$ " model for $\underline{\mathbf{M}}$ iff $\mathbf{A}, \mathbf{B}$, and $\underline{\mathbf{G}}$ constitute a conjunctive "12G" model for $\underline{\mathbf{M}}^{c}$ (for a generic discussion of the duality of disjunctive and conjunctive models, see Leenen, Van Mechelen, \& De Boeck, 1999). Indeed:

$$
m_{i j k}=1 \Leftrightarrow \exists p, q: a_{i p}=1 \wedge b_{j q}=1 \wedge g_{p q k}=1
$$

is equivalent to

$$
\begin{aligned}
m_{i j k}^{c}=1 & \Leftrightarrow \neg\left(\exists p, q: a_{i p}=1 \wedge b_{j q}=1 \wedge g_{p q k}=1\right) \\
& \Leftrightarrow \forall p, q: a_{i p}=0 \vee b_{j q}=0 \vee g_{p q k}=0 .
\end{aligned}
$$

The duality implies that the uniqueness theorem for the disjunctive Tucker2-HICLAS model also implies a sufficient condition for the uniqueness of a conjunctive Tucker2-HICLAS model. 


\subsubsection{Graphical Representation}

A comprehensive graphical representation of the conjunctive Tucker2-HICLAS model is not readily available. Yet, one may consider two alternatives, each with specific advantages and disadvantages. As a first alternative, given the duality of disjunctive and conjunctive models, one may graphically represent $\mathbf{A}, \mathbf{B}$, and $\underline{\mathbf{G}}$ as if they constituted a disjunctive model. This graphical representation has the advantage of being comprehensive and the disadvantage that the association relation can only be derived via exclusion: an object $i$ is associated with attribute $j$ according to source $k$ iff no downward path exists from object $i$ to attribute $j$ that includes source $k$.

As a second alternative, for each object $i$ (object class), one may draw a separate picture from which the association relation in $\underline{\mathbf{M}}_{i::}$ can be derived in a direct way. To this aim, one graphically represents the matrices $\tilde{\mathbf{A}}^{(i)}$ and $\mathbf{B}$ (with $\tilde{\mathbf{A}}^{(i)}$ defined by (14)) as a conjunctive twoway HICLAS model. More specifically, a double box is drawn for each of the $2^{Q}$ bundle patterns, with the upper box containing the corresponding sources and the lower box the corresponding attributes; empty boxes are hatched. Subsequently, the hierarchical relations are indicated by lines between the double boxes. Note that $\mathbf{B}$ does not necessarily represent the relations of equivalence and hierarchy among the attributes in $\underline{\mathbf{M}}_{i::}$. Yet, as we are interested primarily in the hierarchical classification of the attributes in $\underline{\mathbf{M}}$, we prefer to keep $\mathbf{B}$ constant across objects (note that the hierarchical classifications of the objects and sources have to be represented in a separate picture). From the conjunctive HICLAS representations, the association relation can be derived as follows: Object $i$ is associated with Attribute $j$ according to Source $k$ iff $j$ is below $k$ in the representation for $i$. For example, from the representations in Figure 3, it can be read that "Annie" is suitable for the job of "Shop assistant" according to Rater A, because "Shop assistant" is below Rater $A$ in the representation for "Annie." The latter representation has the advantage of being easily interpretable and the disadvantage that the representation of the association relation is spread over multiple pictures.

\section{Algorithm}

Since a $(P, Q)$ Tucker2-HICLAS model is a $(P, Q, R)$ Tucker3-HICLAS model with $R \leq$ $\min (K, P Q)$, it is clear that a $(P, Q)$ Tucker2-HICLAS model can be estimated by means of the Tucker3-HICLAS algorithm. For example, one could estimate a $(P, Q, R)$ Tucker3-HICLAS model with $R=\min (K, P Q)$ and sum over $r$ in the Tucker3-HICLAS association rule (4). However, for reasons of computational efficiency (i.e., estimating three arrays instead of four), we developed a separate Tucker2-HICLAS algorithm. Note that the Tucker2-HICLAS algorithm was initially developed for fitting disjunctive Tucker2-HICLAS models; however, given the duality of the disjunctive and conjunctive models, it can be used to obtain conjunctive Tucker2-HICLAS models as well.

Given a binary $I \times J \times K$ data array $\underline{\mathbf{D}}$ and a rank $(P, Q)$, the Tucker2-HICLAS algorithm aims at finding a binary $I \times J \times K$ model array $\underline{\mathbf{M}}$ that can be represented by a $(P, Q)$ Tucker2HICLAS model and that minimizes the loss function

$$
f(\underline{\mathbf{M}})=\sum_{i=1}^{I} \sum_{j=1}^{J} \sum_{k=1}^{K}\left(d_{i j k}-m_{i j k}\right)^{2} .
$$

Since $\underline{\mathbf{D}}$ and $\underline{\mathbf{M}}$ are binary, (19) can be considered both a least squares and a least absolute deviations loss function.

As with all hierarchical classes algorithms, the algorithm successively executes two main routines. In the first routine, it looks by means of an alternating least squares procedure for arrays $\mathbf{A}, \mathbf{B}$, and $\underline{\mathbf{G}}$ that combine by (7) to $\underline{\mathbf{M}}$ for which (19) is minimal. The first routine starts from an 


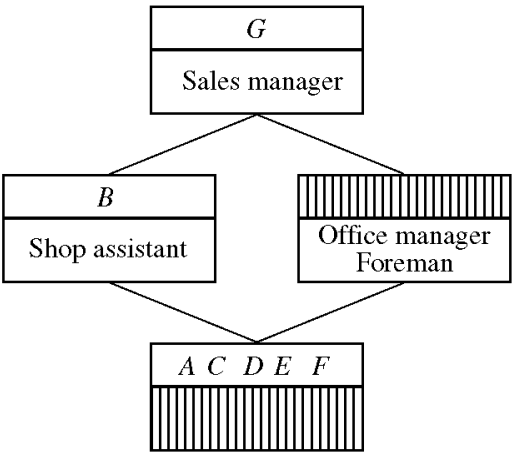

Martha

Rose

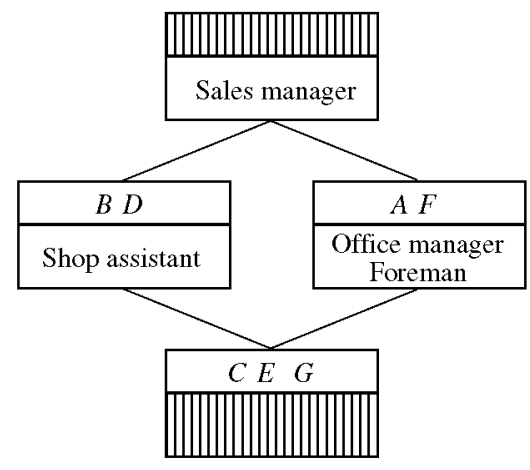

Tom

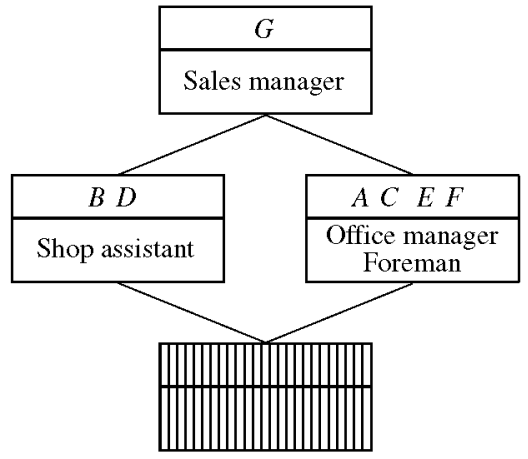

Bob

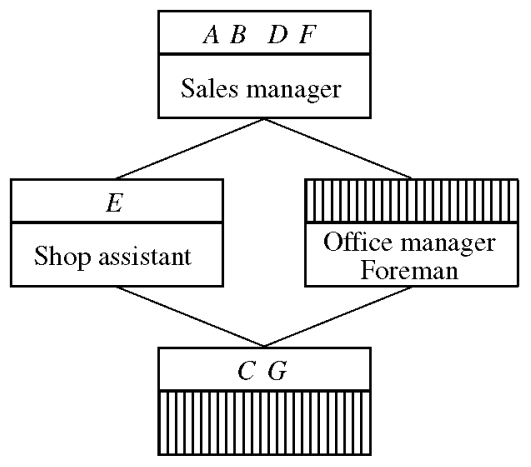

John

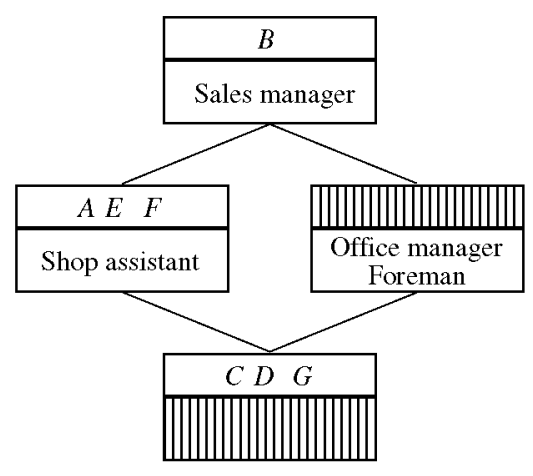

Annie

FIGURE 3.

Type 2 graphical representation of the conjunctive Tucker2-HICLAS model in Table 4. 
initial configuration for the bundle matrices. Such an initial configuration may, for instance, be obtained by performing HICLAS analyses (De Boeck et al., 1988) on the matricized $I \times J K$ and $J \times I K$ data array $\underline{D}$ in ranks $P$ and $Q$ respectively, which yield an object bundle matrix of rank $P$ and an attribute bundle matrix of rank $Q$. Assuming an initial configuration $\mathbf{A}^{(0)}, \mathbf{B}^{(0)}$, the optimal core array $\underline{\mathbf{G}}^{(0)}$, which minimizes (19), is calculated conditionally upon $\mathbf{A}^{(0)}$ and $\mathbf{B}^{(0)}$. In the next steps, $\mathbf{A}^{(w)}$ is re-estimated conditionally upon $\mathbf{B}^{(w-1)}$ and $\underline{\mathbf{G}}^{(w-1)} ; \mathbf{B}^{(w)}$ conditionally upon $\mathbf{A}^{(w)}$ and $\underline{\mathbf{G}}^{(w-1)}$; and $\underline{\mathbf{G}}^{(w)}$ conditionally upon $\mathbf{A}^{(w)}$ and $\mathbf{B}^{(w)}(w=1,2, \ldots)$. This alternating least squares procedure continues until no further improvement in the loss function (19) is observed.

For the conditional estimation of the bundle matrices and the core array, a separability property of the loss function (19) can be used (Chaturvedi \& Carroll, 1994). This property implies that the contribution of the bundle pattern of an object $i, a_{i .}$, to the loss function (19) can be separated from the contribution of the bundle patterns of other objects. Hence, an optimal estimate of $\mathbf{A}$ (conditional upon $\mathbf{B}$ and $\underline{\mathbf{G}}$ ) can be obtained by consecutively estimating the bundle patterns of the $I$ objects; the latter can be done by means of Boolean regression (Leenen \& Van Mechelen, 1998). Similarly, conditionally optimal estimates of $\mathbf{B}$ and $\mathbf{G}$ can be obtained by consecutively optimizing the bundle patterns of the $J$ attributes and the core planes of the $K$ sources.

The $\mathbf{A}, \mathbf{B}$, and $\underline{\mathbf{G}}$ that are obtained at the end of the first routine are only restricted to represent the association relation in $\underline{\mathbf{M}}$. Therefore, in the second routine, $\mathbf{A}, \mathbf{B}$, and $\underline{\mathbf{G}}$ are transformed so as to make them represent the relations of equivalence and hierarchy as well. To this aim, a closure operation is successively applied to $\mathbf{A}, \mathbf{B}$, and $\underline{\mathbf{G}}$, implying that zero-entries of $\mathbf{A}, \mathbf{B}$, and $\underline{\mathbf{G}}$ are turned into one if this change does not alter $\underline{\mathbf{M}}$ (nor, then, the value on the loss function). Note that this closure operation is a sufficient though not necessary condition for the representation of the equivalence and hierarchical relations in the three modes of $\underline{\mathbf{M}}$.

\section{Simulation Study}

In this section, we briefly discuss the main results of a simulation study performed to evaluate the Tucker2-HICLAS algorithm. More specifically, goodness of fit (i.e., how well the algorithm succeeds in minimizing the loss function) and goodness of recovery (i.e., how well the algorithm succeeds in recovering the underlying truth) of Tucker2-HICLAS solutions were examined.

\subsection{Design and Procedure}

Three different types of binary $I \times J \times K$ arrays are involved in the evaluation of the algorithm: a true array $\underline{\mathbf{T}}$, which can be represented by a disjunctive $(P, Q)$ Tucker2-HICLAS model; a data array $\underline{\mathbf{D}}$, which is $\underline{\mathbf{T}}$ perturbed with error; and the model array $\underline{\mathbf{M}}$ yielded by the algorithm, which can also be represented by a disjunctive $(P, Q)$ Tucker2-HICLAS model.

Three parameters were systematically varied in a complete trifactorial design:

(a) the Size, $I \times J \times K$, of $\underline{\mathbf{T}}, \underline{\mathbf{D}}$, and $\underline{\mathbf{M}}$, at 4 levels: $15 \times 15 \times 15,10 \times 20 \times 20,30 \times 20 \times 10$, and $30 \times 20 \times 60$;

(b) the True rank, $(P, Q)$, of the Tucker2-HICLAS model for $\underline{\mathbf{T}}$, at 9 levels: $(2,2),(2,3),(2,4)$, $(3,2),(3,3),(3,4),(4,2),(4,3)$, and $(4,4)$;

(c) and the Error level, $\varepsilon$, which is the proportion of discrepancies between $\underline{\mathbf{T}}$ and $\underline{\mathbf{D}}$, at 5 levels: $.00, .05, .10, .20$, and .30 .

For each combination of Size $I \times J \times K$, True rank $(P, Q)$, and Error level $\varepsilon, 20$ arrays $\mathbf{A}$, $\mathbf{B}$, and $\underline{\mathbf{G}}$ were generated with entries that were independent realizations of a Bernoulli variable with the parameter value of the distribution fixed at that proportion of ones in $\mathbf{A}, \mathbf{B}$, and $\underline{\mathbf{G}}$ that yields an expected proportion of ones in $\underline{\mathbf{T}}$ of .5. Note that this parameter value differs across the 
levels of True rank. Next, a data array $\underline{\mathbf{D}}$ was constructed from each true array $\underline{\mathbf{T}}$ by randomly altering the value of a proportion $\varepsilon$ of the entries in $\underline{\mathbf{T}}$.

On each of the resulting 3600 data arrays a $(P, Q)$ Tucker2-HICLAS analysis was performed, with $(P, Q)$ equal to the corresponding True rank. Two initial configurations for the bundle matrices $\mathbf{A}$ and $\mathbf{B}$ were obtained by performing HICLAS analyses (De Boeck et al., 1988) on the matricized $I \times J K$ and $J \times I K$ data array $\underline{\mathbf{D}}$ in ranks $P$ and $Q$, respectively; and, alternately, INDCLAS analyses (Leenen et al., 1999) on $\underline{\mathbf{D}}$ in ranks $P$ and $Q$. Both analyses yield an object bundle matrix A of rank $P$ and an attribute bundle matrix B of rank $Q$. In the algorithm's alternating least-squares procedure that starts from one of these two initial configurations, the bundle matrices can be estimated in two different orderings. Hence, we ran the algorithm four times. Out of these four resulting solutions, the solution that minimizes (19) was retained.

Regarding the fulfillment of the specified uniqueness conditions in Subsection 2.2.3, note that $45.1 \%$ of the randomly generated true models fulfill these conditions. Further, Figure 4 shows for each combination of Size and True rank the proportion of randomly generated true models that satisfies these conditions. It is clear that these proportions increase with larger Size and lower True rank. Note, however, that the uniqueness conditions as specified above are sufficient but not necessary, implying that true models that do not satisfy these conditions may still be unique. With respect to the latter, the simulation results for the true models that do not fulfill the sufficient uniqueness conditions show that if $\mathbf{T}=\underline{\mathbf{M}}, 95.4 \%$ of the decompositions obtained from the algorithm are identical to the true decompositions. The latter result suggests, indeed, that other sufficient conditions for uniqueness may exist.

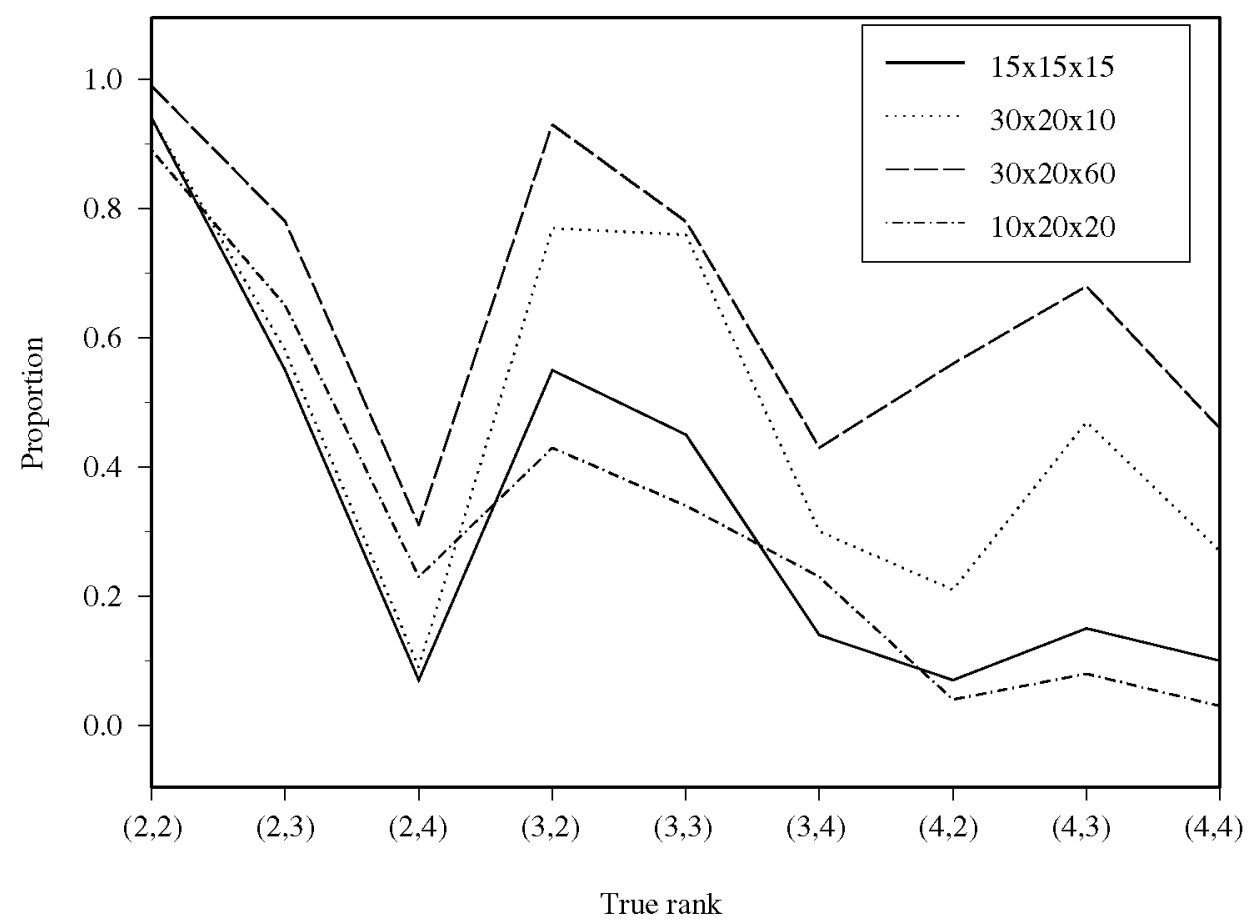

FIGURE 4.

Proportion of randomly generated true models that satisfy the uniqueness conditions at levels of True rank and Size. 


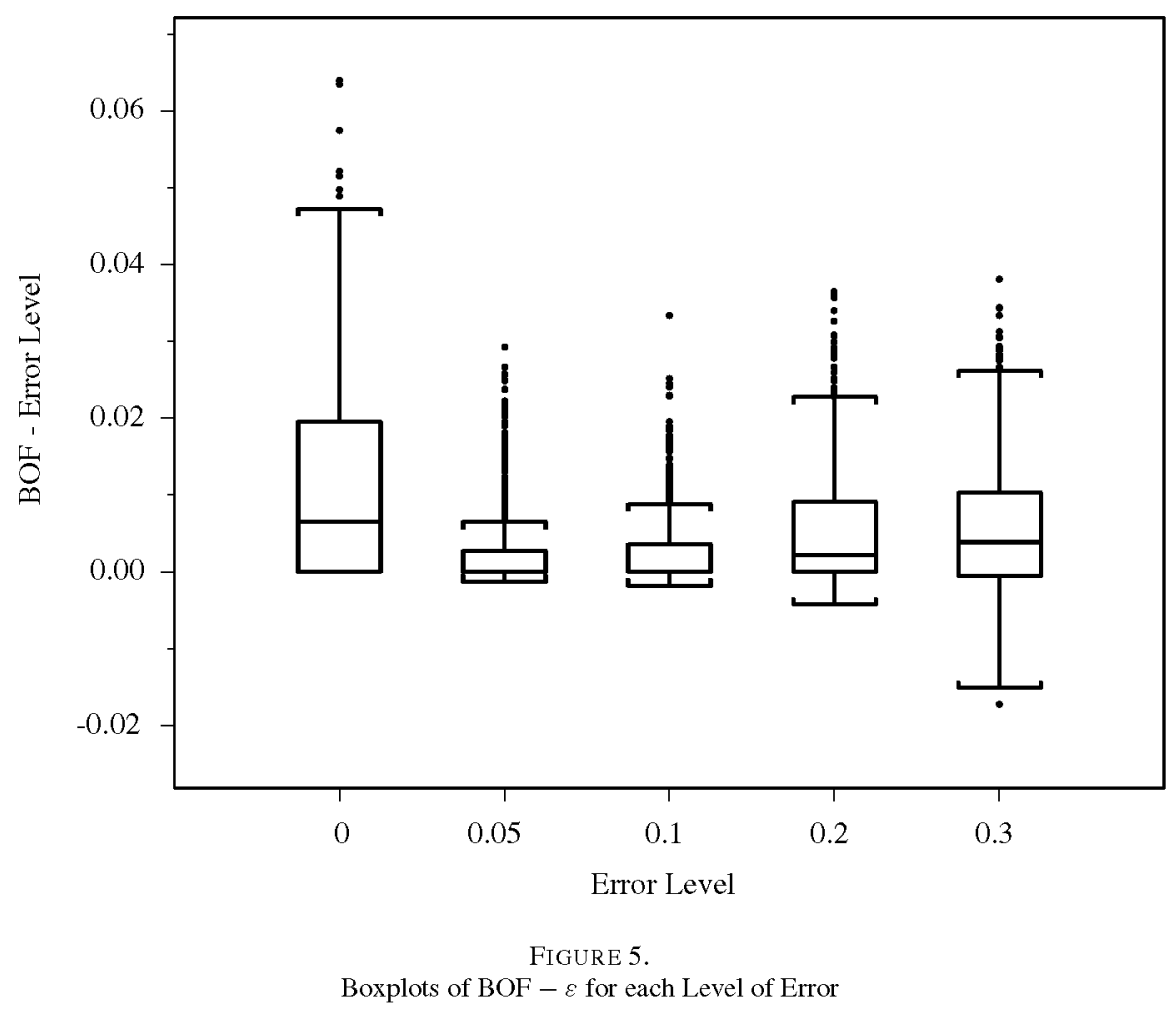

\subsection{Goodness of Fit}

To study how well the Tucker2-HICLAS algorithm succeeds in minimizing the loss function (19), the following badness-of-fit (BOF) statistic was used, which simply gives the proportion of the elements of $\underline{\mathbf{M}}$ that differ from those in $\underline{\mathbf{D}}$ :

$$
\mathrm{BOF}=\frac{\sum_{i=1}^{I} \sum_{j=1}^{J} \sum_{k=1}^{K}\left(d_{i j k}-m_{i j k}\right)^{2}}{I \times J \times K} .
$$

Of the 3600 analyses (5 Error levels $\times 720$ analyses per Error level), 1582 , or $43.9 \%$, ended in a solution with a BOF value smaller than or equal to $\varepsilon$. As the Error level $\varepsilon$ constitutes an upper bound for the BOF of the global minimum, the latter implies that the algorithm ended in a local minimum in a considerable number of cases. An analysis of variance with $\mathrm{BOF}-\varepsilon$ as the dependent variable yielded an intraclass correlation $\hat{\rho}_{I}$ (Haggard, 1958; Kirk, 1982) of .13 for the main effect of Error Level. This main effect implies, on the one hand, that the higher $\varepsilon$, the harder it is for the algorithm to find a model that is as close to the data as the truth is, and, on the other hand, that the simulation results are better for (slightly) error-perturbed data than for error-free data (see Figure 5). Note that the latter unexpected result was also obtained in other hierarchical classes simulation studies. Other effects are not discussed: In this and the following analyses of variance only effects accounting for at least $10 \%$ of the variance of the dependent variable will be considered (i.e., $\hat{\rho}_{I} \geq .10$ ).

\subsection{Goodness of Recovery}

Goodness of recovery will be evaluated with respect to (1) the association relation and (2) the equivalence and hierarchical relations. 
(1) The proportion of discrepancies between $\underline{\mathbf{T}}$ and $\underline{\mathbf{M}}$ was calculated as a measure of the badness of recovery (BOR) of the association relation:

$$
\mathrm{BOR}=\frac{\sum_{i=1}^{I} \sum_{j=1}^{J} \sum_{k=1}^{K}\left(t_{i j k}-m_{i j k}\right)^{2}}{I \times J \times K} .
$$

The mean BOR across the 3600 observations equals .022 , implying that the model yielded by the algorithm differs on average $2.2 \%$ from the underlying truth. An analysis of variance with BOR as the dependent variable yields a main effect of Error level $\left(\hat{\rho}_{I}=.53\right)$ : Except for error-free data, badness of recovery clearly increases with higher Error levels (see Figure 6a). Furthermore, the True rank $\times$ Error level interaction $\left(\hat{\rho}_{I}=.12\right)$ has to be taken into account, indicating that the effect of Error level increases with higher True rank (see Figure 6b).

(2) To assess the recovery of the relations of equivalence and hierarchy, we define the object equivalence and hierarchy matrix associated with $\underline{\mathbf{M}}$ (resp. $\underline{\mathbf{T}}$ ) as the $I \times I$ binary matrix $\mathbf{U}^{(M)}$ (resp. $\left.\mathbf{U}^{(\mathbf{T})}\right)$, with $u_{i i^{\prime}}^{(\mathbf{M})}=1$ (resp. $u_{i i^{\prime}}^{(\mathbf{T})}=1$ ) iff object $i$ is equivalent to or hierarchically lower than object $i^{\prime}$ in $\underline{\mathbf{M}}$ (resp. $\underline{\mathbf{T}}$ ). Subsequently, the proportion of discrepancies between $\mathbf{U}^{\mathbf{T}}$ and $\mathbf{U}^{\mathbf{M}}$ was calculated yielding a badness-of-equivalence-and-hierarchy-recovery (BOEHR) statistic for the objects:

$$
\text { BOEHR }=\frac{\sum_{i=1}^{I} \sum_{i^{\prime}=1}^{I}\left(u_{i i^{\prime}}^{(\mathbf{T})}-u_{i i^{\prime}}^{(\mathbf{M})}\right)^{2}}{I^{2}} .
$$

A BOEHR statistic for the attributes and sources was defined similarly.

The mean value on BOEHR across the 3600 observations equals $.040, .039$, and .054 , for the objects, attributes, and sources, respectively, implying that, on average, $96 \%, 96.1 \%$, and $94.6 \%$ of the true equivalence and hierarchical relations are recovered. An analysis of variance with BOEHR as the dependent variable shows a main effect of Error level ( $\hat{\rho}_{I}$ amounts to .26, .30 , and .21 for objects, attributes, and sources, respectively): Apart from error-free data, the recovery deteriorates with increasing Error level (see Figure 7).

\subsection{Conclusion and Discussion}

With respect to goodness of fit, we may conclude that the algorithm ends in more than half of the cases in a local minimum. The incidence of local minima depends mainly on the amount of error in the data. On the one hand, the number of local minima increases with higher Error levels, but, on the other hand, the local minima problem is also particularly severe in case of error-free data. A further investigation of the latter unexpected finding showed that it is linked to the quality of the initial HICLAS configuration for $\mathbf{A}$ and $\mathbf{B}$, which is worst for error-free data with at least $68.6 \%$ of the initial HICLAS configurations constituting local minima as compared to at least $20 \%$ for the other Error levels. Moreover, for error-free data the algorithm on average performed a lower number of iterations. In general, one may deal with the local minima problem by means of a multistart procedure. More specifically, for a small subset of datasets we found that a multistart procedure that uses initial HICLAS configurations perturbed with a small amount of random error works particularly well.

With respect to goodness of recovery, we may conclude that the algorithm succeeds quite well in recovering the underlying relations of association, equivalence, and hierarchy. Goodness of recovery is mostly influenced by the amount of error on the data: Except for error-free data, the goodness of recovery deteriorates with increasing Error level. Note that this result qualifies the local minima problem, implying that, although the algorithm often ends up in a local minimum, these local minima are quite close to the truth. 


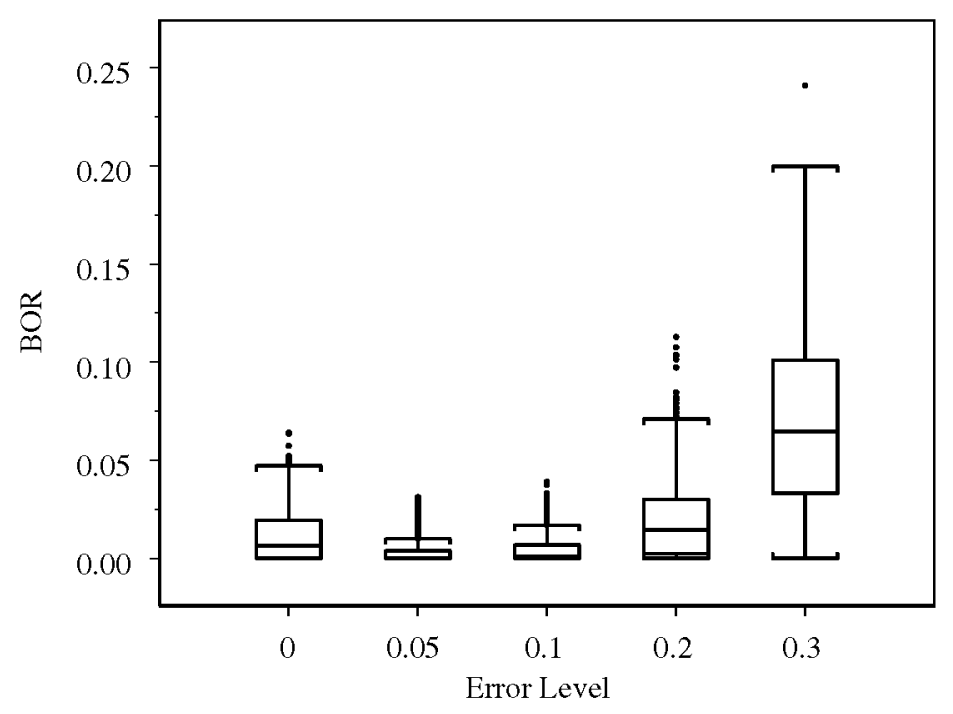

(a) Boxplots for each Level of Error

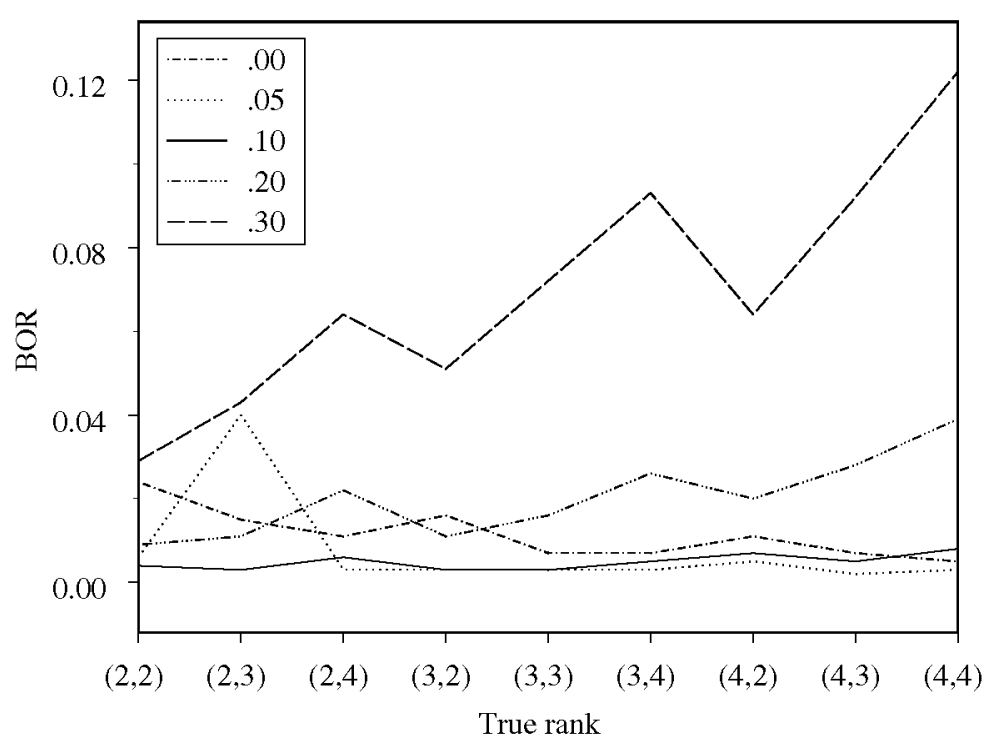

(b) At levels of True rank $\times$ Error

Figure 6.

Badness of Recovery

\section{Illustrative Application}

In this section, we present a Tucker2-HICLAS analysis of data from a longitudinal study on interpersonal emotions. As one research question focuses on tracing the full history of interpersonal emotions, the time mode was not reduced. In this study, a participant was asked to name for each of six role descriptions the person in her life who best fitted the role, with the role descriptions being mother, father, partner, and three unstable relationships. Subsequently, over a period of five months, the participant was asked to indicate every 15 days which of 40 emotions 


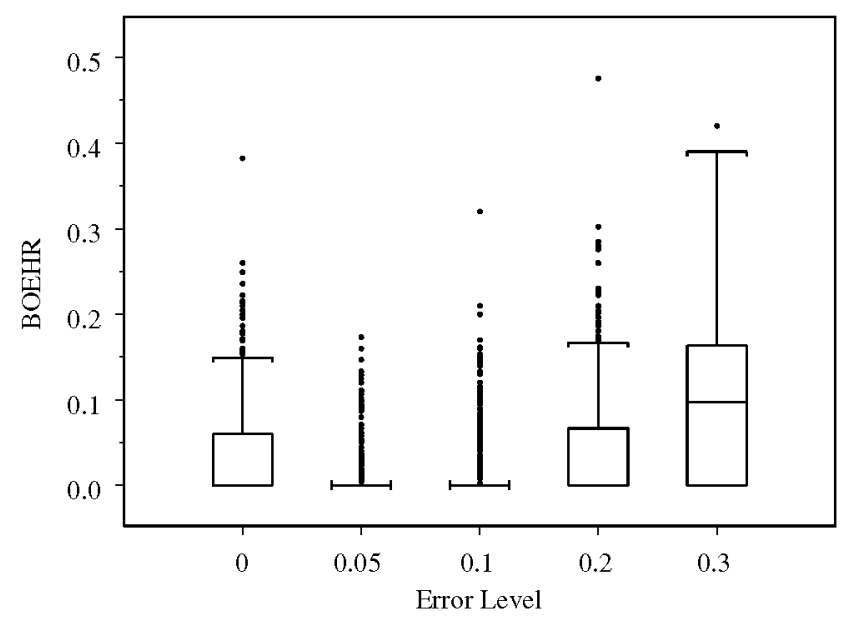

(a) Objects

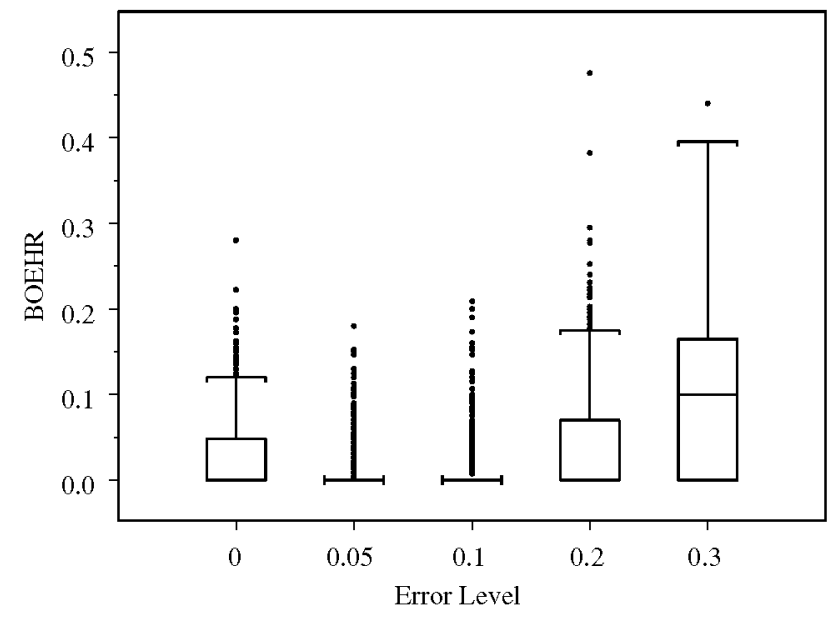

(b) Attributes

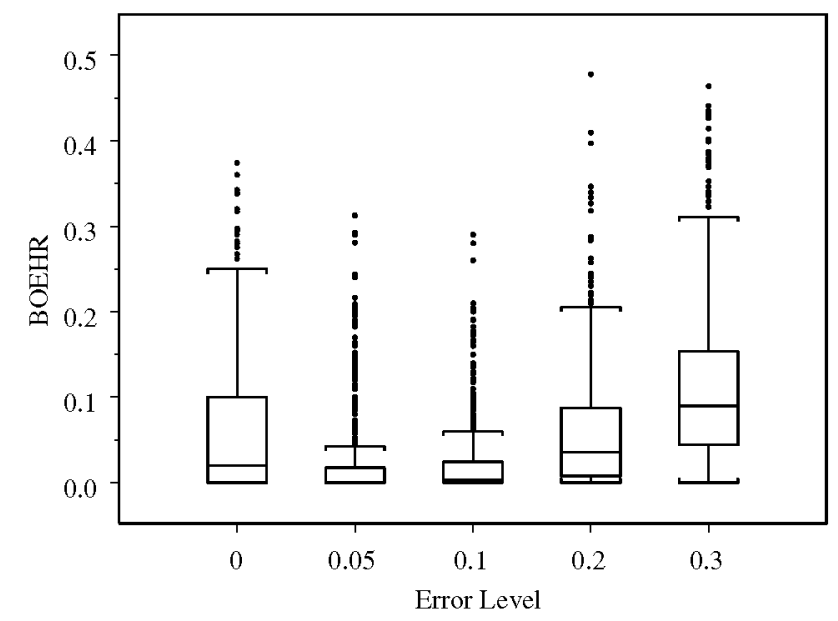

(c) Sources

FIGURE 7.

Boxplots of Badness of Equivalence and Hierarchy Recovery for each Level of Er 
she currently experienced toward the 6 target persons. This resulted in a binary 6 target persons $\times 40$ emotions $\times 10$ time points data array $\underline{\mathbf{D}}$ with $d_{i j k}=1$ if target person $i$ elicited emotion $j$ at time point $k$ and 0 otherwise.

In this application, we will only consider conjunctive analyses. Many emotion theorists argue that complex emotions arise from the conjunction of more basic emotions (see e.g., Plutchik, 1962; Izard, 1977), and, as explained in Subsection 2.3, conjunctive Tucker2-HICLAS models are suitable for tracing such conjunctive dependencies. More specifically, conjunctive " $1{ }^{c} 2 \mathrm{G}$ " (i.e., with the association rule specified by (12)) Tucker2-HICLAS models in ranks $(1,1)$ through $(5,5)$ were fitted to $\underline{\mathbf{D}}$. To select a model out of the 25 resulting models, we made use of a pseudobinomial selection heuristic, which outperformed other selection heuristics in a simulation study (Leenen, Van Mechelen, \& De Boeck, 2001; Leenen \& Van Mechelen, 2001): First, for each model the sum $s$ of target and emotion bundles, and the BOF-value (20) were calculated. Subsequently, for each value of $s$, only the best fitting model was retained (see also Timmerman \& Kiers, 2000). Finally, the pseudo-binomial rule selects the smallest $s$ for which $\mathrm{BOF}_{s+1} \times n$ exceeds the first decile of the binomial distribution $\operatorname{Bin}\left(n, \mathrm{BOF}_{s}\right)$, where $n$ is the number of entries in $\underline{\mathbf{D}}$ and $\mathrm{BOF}_{s}$ denotes the $\mathrm{BOF}$-value of the retained model with sum of bundles $s$. Table 5 shows that applying the latter rule to the 25 obtained models for $\underline{\mathbf{D}}$ results in the selection of the $(4,3)$ model. This model, which is represented by $\mathbf{A}, \mathbf{B}$, and $\mathbf{G}$ in Tables 6 and 7 , has a BOF-value

TABLE 5 .

$\mathrm{BOF}$ and pseudo-binomial rule statistic of the conjunctive Tucker2-HICLAS models for the interpersonal emotion data as a function of the sum of bundles $s$

\begin{tabular}{rccc}
\hline$s$ & Rank & BOF & $P\left[X \leq\left(n \times \mathrm{BOF}_{s+1}\right) \mid X \sim \operatorname{Bin}\left(n, \mathrm{BOF}_{s}\right)\right]$ \\
\hline 2 & $(1,1)$ & .263 & .097 \\
3 & $(2,1)$ & .252 & .000 \\
4 & $(2,2)$ & .209 & .003 \\
5 & $(3,2)$ & .186 & .000 \\
6 & $(3,3)$ & .158 & .006 \\
7 & $(4,3)$ & .139 & .131 \\
8 & $(4,4)$ & .131 & .003 \\
9 & $(5,4)$ & .112 & .003 \\
10 & $(5,5)$ & .095 & \\
\hline
\end{tabular}

TABLE 6.

Core array of the conjunctive $(4,3)$ Tucker2-HICLAS model for the interpersonal emotion data

\begin{tabular}{|c|c|c|c|c|c|c|c|c|c|c|c|c|}
\hline \multirow[b]{2}{*}{ Time points } & \multicolumn{4}{|c|}{$E B_{1}$} & \multicolumn{4}{|c|}{$E B_{2}$} & \multicolumn{4}{|c|}{$E B_{3}$} \\
\hline & $T B_{1}$ & $T B_{2}$ & $T B_{3}$ & $T B_{4}$ & $T B_{1}$ & $T B_{2}$ & $T B_{3}$ & $T B_{4}$ & $T B_{1}$ & $T B_{2}$ & $T B_{3}$ & $T B_{4}$ \\
\hline Time point 1 & 0 & 1 & 0 & 1 & 0 & 0 & 1 & 0 & 1 & 0 & 0 & 1 \\
\hline Time point 2 & 0 & 1 & 0 & 1 & 0 & 0 & 1 & 0 & 1 & 0 & 0 & 1 \\
\hline Time point 3 & 0 & 1 & 0 & 0 & 0 & 0 & 1 & 0 & 1 & 0 & 0 & 1 \\
\hline Time point 4 & 0 & 1 & 0 & 1 & 0 & 0 & 1 & 0 & 1 & 0 & 0 & 1 \\
\hline Time point 5 & 0 & 1 & 0 & 1 & 0 & 0 & 1 & 0 & 1 & 0 & 0 & 1 \\
\hline Time point 6 & 0 & 0 & 0 & 1 & 0 & 1 & 1 & 0 & 1 & 0 & 0 & 1 \\
\hline Time point 7 & 0 & 1 & 1 & 1 & 0 & 0 & 0 & 0 & 1 & 0 & 1 & 0 \\
\hline Time point 8 & 0 & 1 & 1 & 0 & 0 & 0 & 0 & 1 & 1 & 0 & 1 & 0 \\
\hline Time point 9 & 0 & 1 & 1 & 0 & 0 & 0 & 0 & 1 & 1 & 0 & 1 & 0 \\
\hline Time point 10 & 0 & 1 & 1 & 1 & 0 & 0 & 0 & 0 & 1 & 0 & 0 & 0 \\
\hline
\end{tabular}


TABLE 7.

Target and emotion bundle matrices of the conjunctive $(4,3)$ Tucker2-HICLAS model for the interpersonal emotion data

\begin{tabular}{|c|c|c|c|c|c|c|c|c|}
\hline \multicolumn{5}{|c|}{ Target bundle matrix } & \multicolumn{4}{|c|}{ Emotion bundle matrix } \\
\hline Targets & $T B_{1}$ & $T B_{2}$ & $T B_{3}$ & $T B_{4}$ & Emotions & $E B_{1}$ & $E B_{2}$ & $E B_{3}$ \\
\hline Unstable relationship 2 & 1 & 1 & 1 & 0 & Dislike & 1 & 1 & 1 \\
\hline Unstable relationship 3 & 1 & 1 & 0 & 1 & Disgust & 1 & 1 & 1 \\
\hline Mother & 1 & 0 & 1 & 1 & Hate & 1 & 1 & 1 \\
\hline Father & 1 & 0 & 1 & 1 & Superior & 1 & 1 & 1 \\
\hline Partner & 0 & 1 & 1 & 1 & Bored & 1 & 1 & 1 \\
\hline Unstable relationship 1 & 0 & 1 & 1 & 1 & Secure & 1 & 1 & 0 \\
\hline & & & & & A bond & 1 & 1 & 0 \\
\hline & & & & & Warmth & 1 & 1 & 0 \\
\hline & & & & & Detached & 1 & 0 & 1 \\
\hline & & & & & Uncomfortable & 1 & 0 & 1 \\
\hline & & & & & Close & 1 & 0 & 1 \\
\hline & & & & & Upset & 1 & 0 & 1 \\
\hline & & & & & Confused & 1 & 0 & 1 \\
\hline & & & & & Nervous & 1 & 0 & 1 \\
\hline & & & & & Angry & 1 & 0 & 1 \\
\hline & & & & & Frustrated & 1 & 0 & 1 \\
\hline & & & & & Defensive & 1 & 0 & 1 \\
\hline & & & & & Pity & 0 & 1 & 1 \\
\hline & & & & & Concern & 0 & 1 & 1 \\
\hline & & & & & Jealous & 1 & 0 & 0 \\
\hline & & & & & Fine & 0 & 1 & 0 \\
\hline & & & & & Like & 0 & 1 & 0 \\
\hline & & & & & Happy & 0 & 1 & 0 \\
\hline & & & & & At ease & 0 & 1 & 0 \\
\hline & & & & & Easy & 0 & 1 & 0 \\
\hline & & & & & Joy & 0 & 1 & 0 \\
\hline & & & & & Comfortable & 0 & 1 & 0 \\
\hline & & & & & Interest & 0 & 1 & 0 \\
\hline & & & & & Confident & 0 & 1 & 0 \\
\hline & & & & & Intelligent & 0 & 1 & 0 \\
\hline & & & & & Admiration & 0 & 1 & 0 \\
\hline & & & & & Respect & 0 & 1 & 0 \\
\hline & & & & & Inferior & 0 & 0 & 1 \\
\hline & & & & & Rejected & 0 & 0 & 1 \\
\hline & & & & & Sorrow & 0 & 0 & 1 \\
\hline & & & & & Sad & 0 & 0 & 1 \\
\hline & & & & & Oppressed & 0 & 0 & 1 \\
\hline & & & & & Fear & 0 & 0 & 1 \\
\hline & & & & & Friendly & 0 & 0 & 0 \\
\hline & & & & & Love & 0 & 0 & 0 \\
\hline
\end{tabular}

of .139 and a Jaccard Goodness-of-fit value of .748. Note that $\mathbf{A}^{c}, \mathbf{B}$, and $\mathbf{G}$ fulfill the sufficient uniqueness conditions that were specified in Subsection 2.2.3.

The selected $(4,3)$ model reduces the 40 emotions to three emotion bundles, implying that eight different emotion bundle patterns may occur. From Table 7 it appears that all eight possible bundle patterns show up. The corresponding eight emotion classes, labeled $E_{1}$ through $E_{8}$, and their hierarchical interrelations, which may also be derived from Table 7 , are graphically repre- 


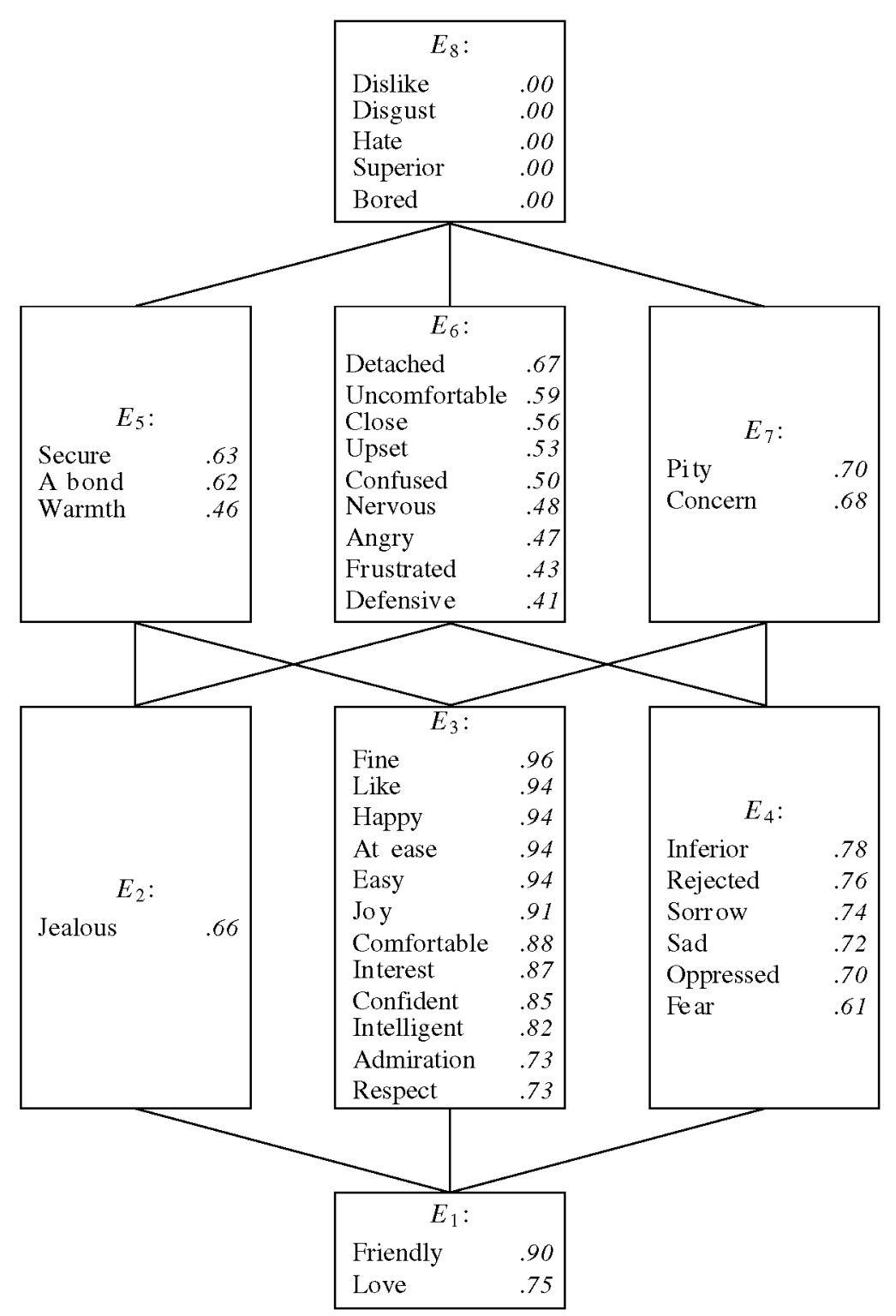

FIGURE 8

Emotion structure of the interpersonal emotion data and goodness-of-fit values for all emotions.

sented in Figure 8; the classes also include the goodness-of-fit values of all single emotions. As is often the case for conjunctive hierarchical classes models, the hierarchically lowest class; that is, $E_{8}$ contains emotions that do not fit in the model; this class will therefore not be included in our further interpretation. The emotion structure appears to have an evaluative basis: The emotions in $E_{1}, E_{3}$ and $E_{5}$ all have a positive valence, whereas the emotions in $E_{2}, E_{4}$ and $E_{6}$ all have a negative valence. The valence of the emotion terms in $E_{7}$ is rather ambiguous, though. The latter becomes more comprehensible, however, if we note that the emotions from this class (e.g., "pity") emerge from the conjunction of some positive ( $E_{3}$, e.g., "fine") and negative emotions ( $E_{4}$, e.g., "inferior"). Hence, $E_{7}$ possibly includes ambivalent emotions. Further insights may be 
gained from studying other hierarchical relations among emotion classes. For example, a warm and secure bond $\left(E_{5}\right)$ implies jealousy $\left(E_{2}\right)$, which reveals a possible problem for our participant.

The target bundle matrix $\mathbf{A}$ in Table 7 reveals that there are four different target bundle patterns and, hence, four different target classes. Figure 9 shows the conjunctive HICLAS representations for these four target classes, with the emotion classes being labeled in terms of the best fitting emotions. As explained in Subsection 2.3.4, target person $i$ elicits emotion $j$ at time point $k$ iff emotion $j$ is below time point $k$ in the representation for target $i$. Figure 5 can be given the following substantive interpretation: The first unstable relationship and partner relationship are described in generally positive terms ("fine," "secure," "friendly," "jealous") at every time point. Mother and father elicit ambivalence ("pity," "fine," "inferior," "jealous") throughout the study, except for time point 6, where the relationship with the parents goes through a temporary crisis evoking mostly negative emotions ("detached," "inferior," "jealous," "friendly"). The second unstable relationship is very unstable indeed: This relationship first goes from rather positive ("fine," "friendly") over generally positive to rather positive again; then from ambivalent, over mostly negative, to ambivalent again. Finally, the third unstable relationship elicits mostly negative emotions at the first six time points, then becomes rather positive for three time points and ends in ambivalence at time point 10. One may conclude that the Tucker2-HICLAS methodology allows for a very detailed modeling of how relationships develop over time.

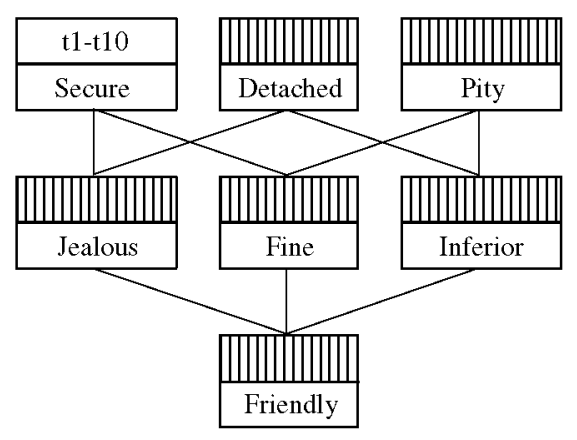

Unstable relationship 1 Partner

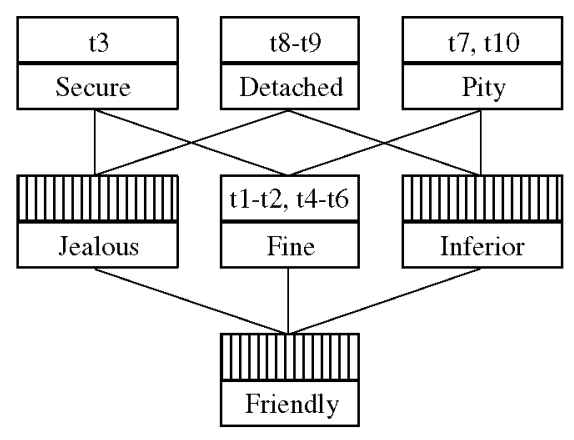

Unstable relationship 2
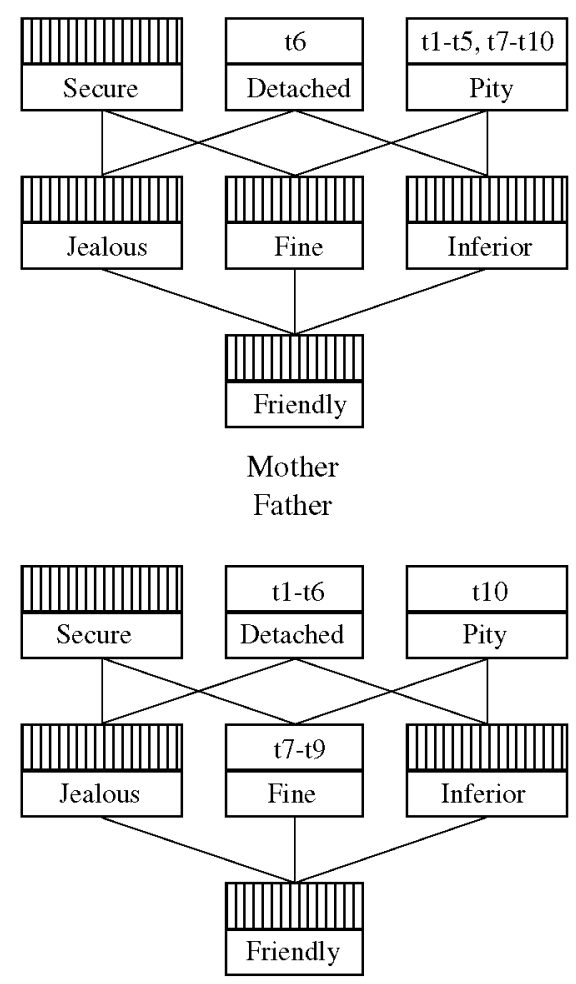

Unstable relationship 3

FIGURE 9.

Type 2 graphical representation of the conjunctive Tucker2-HICLAS model for the interpersonal emotion data. 


\section{Discussion}

In this paper, we presented a new three-way three-mode hierarchical classes model, called Tucker2-HICLAS. The relationship between Tucker2-HICLAS and Tucker3-HICLAS, as outlined in Subsection 2.2.5, is simple, yet perhaps difficult to grasp cognitively. In fact, it holds that given a rank $(P, Q)$, the sets of $(P, Q, R)$ Tucker3-HICLAS models that are obtained by letting $R$ range from 1 through $P Q$ constitute a partition of the set of $(P, Q)$ Tucker2-HICLAS models. In particular, each $(P, Q)$ Tucker2-HICLAS model is a $(P, Q, R)$ Tucker3-HICLAS model, with $R$ equal to the Schein rank (decomposition rank) of the matricized $P Q \times K$ Tucker2-HICLAS core array. Furthermore, given a rank $(P, Q, R)$, the set of $(P, Q, R)$ Tucker3-HICLAS models constitutes a subset of the set of $(P, Q)$ Tucker2-HICLAS models; in particular, the set of $(P$, $Q, R)$ Tucker3-HICLAS models is equal to the subset of $(P, Q)$ Tucker2-HICLAS models for which it holds that the matricized $P Q \times K$ core array has Schein rank $R$. Although as far as we know this has never been discussed before, a similar relation holds between the real-valued Tucker 3 and Tucker 2 models, in which case the concept of Schein rank is simply to be replaced by the ordinary rank concept. In particular, given numbers of components $P, Q$ and $R$, the set of $(P, Q, R)$ Tucker3 models equals the subset of $(P, Q)$ Tucker2 models for which the matricized $P Q \times K$ core arrays have rank $R$.

Despite the close relationship between Tucker2-HICLAS and Tucker3-HICLAS, Tucker2HICLAS has some merits of its own. First, Tucker2-HICLAS minimally reduces one of the three modes; hence, the differences among the association patterns of the elements of this mode are maximally retained in the model. Because of the hierarchical classification of the free mode, the latter is achieved in an insightful way, even when the free mode consists of a huge number of elements. Second, unlike Tucker3-HICLAS, Tucker2-HICLAS includes only three types of parameters, implying that, among other things, the core array is simpler to interpret; as an example, we refer to the insightful interpretation of the core array of the hypothetical data in Section 2. Note that the Tucker2-HICLAS model includes as few types of parameters as the three-way INDCLAS model (Leenen et al., 1999), but is much more general as, for example, INDCLAS restricts the number of bundles to be equal for all three modes.

\section{A. Appendix}

Theorem. If a $(P, Q)$ disjunctive Tucker2-HICLAS decomposition of an $I \times J \times K$ binary array $\underline{\mathbf{M}}$ exists, such that (1) all bundle-specific classes of $\mathbf{A}$ and $\mathbf{B}$ are nonempty, and (2) no object (resp. attribute) plane of $\underline{\mathbf{G}}$ is a subset of the Boolean sum of the other object (resp. attribute) planes of $\underline{\mathbf{G}}$, this decomposition is unique upon a permutation of the object and attribute bundles.

Proof. Assume, without loss of generality, that the first $P$ and $Q$ rows of $\mathbf{A}$ and $\mathbf{B}$, respectively, are elements of the bundle-specific classes. In particular, for $1 \leq i \leq P$, we assume $a_{i p}=1$ iff $p=i$; similarly, for $1 \leq j \leq Q, b_{j q}=1$ iff $q=j$. We will first prove the uniqueness of $\mathbf{A}$ (the proof is similar for $\mathbf{B}$ ) and next the uniqueness of $\mathbf{G}$. The Tucker2-HICLAS decomposition rule (6) implies that the following disjunctive HICLAS decomposition of the matricized $I \times J K$ array $\underline{\mathbf{M}}$ exists:

$$
m_{i(j k)}=\bigoplus_{p=1}^{P} a_{i p} \tilde{b}_{(j k) p},
$$

where

$$
\tilde{b}_{(j k) p}=\bigoplus_{q=1}^{Q} b_{j q} g_{p q k}
$$


is an entry of the $J K \times P$ (attributes $\times$ sources) bundle matrix $\tilde{\mathbf{B}}$. Ceulemans and Van Mechelen (2003) have stated that a disjunctive HICLAS decomposition is unique (upon a permutation of the bundles) if the bundle-specific classes of $\mathbf{A}$ and $\tilde{\mathbf{B}}$ are nonempty. For $\mathbf{A}$, the latter is obvious. To show the latter for $\tilde{\mathbf{B}}$, we first note that the assumption about $\mathbf{B}$ implies that the submatrix $\tilde{\mathbf{B}}^{*}$ based on the first $Q$ rows of $\mathbf{B}$ equals the matricized $Q K \times P$ core array $\underline{\mathbf{G}}$, that is, for all $1 \leq j \leq Q \tilde{b}_{(j k) p}=g_{p j k}$. The nonemptiness of the bundle-specific classes of $\tilde{\mathbf{B}}^{*}$ and, hence, of $\tilde{\mathbf{B}}$, further follows from the restriction on $\underline{\mathbf{G}}$ that no object core plane is a subset of the Boolean sum of the other object core planes. For, this restriction implies that for any object bundle $p^{*}$ $\left(p^{*}=1 \ldots P\right.$ ) an attribute bundle $q$ and a source $k$ exist such that $g_{p q k}=1$ iff $p=p^{*}$. With respect to the core array $\underline{\mathbf{G}}$, the assumption about $\mathbf{A}$ and $\mathbf{B}$ implies that for all $1 \leq i \leq P$ and $1 \leq j \leq Q, m_{i j k}=g_{i j k}$. The latter means that no core entry can be modified without modifying $\underline{\mathbf{M}}$ and, therefore, that $\underline{\mathbf{G}}$ is unique.

\section{References}

Carroll, J.D., \& Chang, J.J. (1970). Analysis of individual differences in multidimensional scaling via an $n$-way generalization of "Eckart-Young" decomposition. Psychometrika, 35, 283-319.

Ceulemans, E., \& Van Mechelen, I. (2003). Uniqueness of $n$-way $n$-mode hierarchical classes models. Journal of Mathematical Psychology, 47, 259-264.

Ceulemans, E., Van Mechelen, I., \& Leenen, I. (2003). Tucker3 hierarchical classes analysis. Psychometrika, 68, 413433.

Chaturvedi, A., \& Carroll. J.D. (1994). An alternating combinatorial optimization approach to fitting the INDCLUS and generalized INDCLUS models. Joumal of Classification, 11, 155-170.

De Boeck, P., \& Rosenberg, S. (1988). Hierarchical classes: Model and data analysis. Psychometrika, 53, 361-381.

Haggard, E.A. (1958). Intraclass correlation and the analysis of variance. New York: Dryden.

Harshman, R.A. (1970). Foundations of the PARAFAC procedure: Models and conditions for an explanatory multi-modal factor analysis. UCLA Working Papers in Phonetics, 16, 1-84.

Izard, C.E. (1977) Human emotions. New York: Plenum Press.

Kim, K.H. (1982). Boolean matrix theory. New York: Marcel Dekker.

Kirk. R.E. (1982). Experimental design: Procedures for the behavioral sciences (2nd ed.). Belmont, CA: Brooks/Cole.

Kroonenberg, P.M. (1983). Three-mode principal component analysis: Theory and applications. Leiden: DSWO.

Kroonenberg, P.M., \& De Leeuw, J. (1980). Principal component analysis of three-mode data by means of alternating least squares algorithms. Psychometrika, 45, 69-97.

Leenen, I., \& Van Mechelen, I. (1998). A branch-and-bound algorithm for Boolean regression. In I. Balderjahn, R. Mathar, \& M. Schader (Eds.), Data highways and information flooding, a challenge for classification and data analysis (pp. 164-171). Berlin, Germany: Springer-Verlag.

Leenen, I., \& Van Mechelen, I. (2001). An evaluation of two algorithms for hierarchical classes analysis. Journal of Classification, 18, 57-80.

Leenen, I., Van Mechelen, I., \& De Boeck, P. (1999). A generic disjunctive/conjunctive decomposition model for $n$-ary relations. Joumal of Mathematical Psychology, 43, 102-122.

Leenen, I., Van Mechelen, I., De Boeck, P. (2001). Models for ordinal hierarchical classes analysis. Psychometrika, 66, 389-404.

Leenen, I., Van Mechelen, I., De Boeck, P., \& Rosenberg, S. (1999). INDCLAS: A three-way hierarchical classes model. Psychometrika, 64, 9-24.

Plutchik, R. (1962). The emotions: Facts, theories, and a new model. New York: Random House.

Timmerman, M.E., \& Kiers, H.A.L. (2000). Three-mode principal components analysis: Choosing the numbers of components and sensitivity to local optima. British Journal of Mathematical and Statistical Psychology, 53, 1-16.

Tucker, L.R. (1966). Some mathematical notes on three-mode factor analysis. Psychometrika, 31, $279-311$.

Van Mechelen, I., De Boeck, P., \& Rosenberg, S. (1995). The conjunctive model of hierarchical classes. Psychometrika, $60,505-521$.

Manuscript received 1 MAY 2001

Final version received 5 MAY 2003 